\title{
Fast neutrino flavor instability in the neutron-star convection layer of three-dimensional supernova models
}

\author{
Robert Glas $\odot,{ }^{1,2, *}$ H.-Thomas Janka ${ }^{1, \dagger}$ Francesco Capozzi@ ${ }^{3, \$}$ Manibrata Sen, ${ }^{4,5, \S}$ Basudeb Dasgupta, ${ }^{6, \|}$ \\ Alessandro Mirizzi, ${ }^{7,8, \uparrow}$ and Günter Sigl, ${ }^{9, * *}$ \\ ${ }^{1}$ Max-Planck-Institut für Astrophysik, Karl-Schwarzschild-Straße 1, 85748 Garching, Germany \\ ${ }^{2}$ Physik Department, Technische Universität München, \\ James-Franck-Straße 1, 85748 Garching, Germany \\ ${ }^{3}$ Max-Planck-Institut für Physik (Werner-Heisenberg-Institut), \\ Föhringer Ring 6, 80805 München, Germany \\ ${ }^{4}$ Department of Physics, University of California Berkeley, Berkeley, California 94720, USA \\ ${ }^{5}$ Department of Physics and Astronomy, Northwestern University, Evanston, Illinois 60208, USA \\ ${ }^{6}$ Tata Institute of Fundamental Research, Homi Bhabha Road, Mumbai, 400005, India \\ ${ }^{7}$ Dipartimento Interateneo di Fisica "Michelangelo Merlin," Via Amendola 173, 70126 Bari, Italy \\ ${ }^{8}$ Istituto Nazionale di Fisica Nucleare - Sezione di Bari, Via Amendola 173, 70126 Bari, Italy \\ ${ }^{9}$ II. Institute for Theoretical Physics, Hamburg University, \\ Luruper Chaussee 149, 22761 Hamburg, Germany
}

(Received 3 December 2019; accepted 14 January 2020; published 2 March 2020)

\begin{abstract}
It has been speculated for a long time that neutrinos from a supernova ( $\mathrm{SN}$ ) may undergo fast flavor conversions near the collapsed stellar core. We perform a detailed study of this intriguing possibility, for the first time analyzing two time-dependent state-of-the-art three-dimensional (3D) SN models of $9 M_{\odot}$ and $20 M_{\odot}$ from recent papers of Glas et al. Both models were computed with multidimensional three-flavor neutrino transport based on a two-moment solver, and both exhibit the presence of the so-called lepton-number emission self-sustained asymmetry (LESA). The transport solution does not provide the angular distributions of the flavor-dependent neutrino fluxes, which are crucial to track the fast flavor instability. To overcome this limitation, we use a recently proposed approach based on the angular moments of the energy-integrated electron lepton-number distribution up to second order, i.e., angle-energy integrals of the difference between $\nu_{e}$ and $\bar{\nu}_{e}$ phase-space distributions multiplied by corresponding powers of the unit vector of the neutrino velocity. With this method we find the possibility of fast neutrino flavor instability at radii smaller than $20 \mathrm{~km}$, which is well interior to the neutrinosphere where neutrinos are still in the diffusive and near-equilibrium regime. Our results confirm recent observations in a two-dimensional (2D) (axisymmetric) SN model and in 2D and 3D models with a fixed matter background, which were computed with Boltzmann neutrino transport. However, the flavor unstable locations are not isolated points as discussed previously, but thin skins surrounding volumes where $\bar{\nu}_{e}$ are more abundant than $\nu_{e}$. These volumes grow with time and appear first in the convective layer of the proto-neutron star (PNS), where a decreasing electron fraction and high temperatures favor the occurrence of regions with negative neutrino chemical potential. Since the electron fraction remains higher in the LESA dipole direction, where convective lepton-number transport out from the nonconvective PNS core slows down the deleptonization, flavor unstable conditions become more widespread in the opposite hemisphere. This interesting phenomenon deserves further investigation, since its impact on SN modeling and possible consequences for SN dynamics and neutrino observations are presently unclear.
\end{abstract}

DOI: 10.1103/PhysRevD.101.063001

\footnotetext{
rglas@mpa-garching.mpg.de ${ }^{\dagger}$ thj@mpa-garching.mpg.de capozzi@mppmu.mpg.de

${ }^{\S}$ manibrata@berkeley.edu

"bdasgupta@theory.tifr.res.in

१alessandro.mirizzi@ba.infn.it ***enter.sigl@desy.de
}

Published by the American Physical Society under the terms of the Creative Commons Attribution 4.0 International license. Further distribution of this work must maintain attribution to the author(s) and the published article's title, journal citation, and DOI. Funded by the Max Planck Society. 


\section{INTRODUCTION}

The deepest supernova $(\mathrm{SN})$ regions provide a unique laboratory to probe neutrino flavor conversions in a nonlinear regime, where the neutrino evolution is determined mainly by their mutual interactions. Indeed, at distances $r \lesssim \mathcal{O}\left(10^{2}\right) \mathrm{km}$ from the center of the $\mathrm{SN}$, the neutrino density $n_{\nu}$ is so high that it dominates the flavor evolution, leading to self-induced neutrino flavor conversions [1-3]. These have been a topic of intense investigation for over a decade [4-10]. See Refs. [11-14] for recent reviews.

In this context, a peculiar type of self-induced flavor conversions, called "fast" instabilities [4,8,10,15-28], is expected to lead to flavor conversions developing on very short distances, $r \lesssim \mathcal{O}(1) \mathrm{m}$. Fast flavor conversions have been associated with "crossings" in the electronneutrino lepton number (ELN) angular distribution, i.e., with a change of sign in the difference between $\nu_{e}$ and $\bar{\nu}_{e}$ number densities as a function of the emission angle [16,18,24]. Conditions for crossings in the ELN were expected to be possible in the neutrino decoupling region in SN cores, where the different flavors have significantly different angular distributions.

This possibility of fast flavor conversions and their potential effects on $\mathrm{SN}$ dynamics and nucleosynthesis has stimulated several studies to assess the occurrence of fast instabilities in different SN models. A first study in this direction was performed in [29], where a dedicated analysis of the angular distributions of the neutrino radiation field for several spherically symmetric [one-dimensional (1D)] SN simulations has not found any crossing in the ELN near the neutrinosphere. More generally, two- (2D) or threedimensional (3D) models can exhibit a large-scale dipole in the ELN emission, termed lepton-emission self-sustained asymmetry (LESA) [30], which also makes a crossing more likely to occur. In this context, the first analyses of fast instabilities in multidimensional SN models have recently been performed in [31,32]. In [31] the authors extracted three snapshots from numerical data in 2D and 3D SN simulations and looked for ELN crossings in the angular distributions of $\nu_{e}$ and $\bar{\nu}_{e}$. They found favorable conditions in extended regions with the radius of 50-70 km. Then, by a linear stability analysis of the neutrino equations of motion, they identified the strength of this instability for a representative point. However, their neutrino distributions were obtained from neutrino transport calculations done in a postprocessing step, dropping the time dependence of both hydrodynamical and neutrino quantities and ignoring matter motions entirely. Hence they are not fully selfconsistent. Conversely, in [32] the authors used fully selfconsistent simulations in $2 \mathrm{D}$, computing neutrino transport with a multiangle Boltzmann solver coupled to hydrodynamics. Applying linear stability analysis near the neutrinosphere, they found no positive signatures of conversion at least for the spatial points and times studied in their particular model.
Furthermore, in [33] the claim was made that in the preshock region, at $r \simeq \mathcal{O}(100) \mathrm{km}$, the residual coherent neutrino-nucleus scatterings could produce a tiny crossing in the ELN, whose presence has been confirmed by the inspection of various numerical simulations. Despite the smallness of the crossing, according to a stability analysis it would be enough to trigger significant fast conversions. However, for a cautioning argument against overinterpreting results of stability analyses, see [34].

Recently, two publications, based again on the SN models considered in [31,32], reported positive detections of locations of ELN crossings deep inside the proto-neutron star (PNS) when investigating the self-consistent 2D corecollapse simulation of an $11.2 M_{\odot}$ star computed with Boltzmann neutrino transport [35] and 3D Boltzmann neutrino-transport results for a fixed matter background at some instants during the postbounce evolution of 11.2 $M_{\odot}$ and $27 M_{\odot}$ progenitors [36]. Because the diffusive conditions for neutrinos in the deep PNS interior imply that the angular distributions of both $\nu_{e}$ and $\bar{\nu}_{e}$ are nearly isotropic, ELN crossings were found only in regions where the "asymmetry parameter" $\Gamma=n_{\bar{\nu}_{e}} / n_{\nu_{e}}$, i.e., the ratio of the number densities of both neutrino types, is close to unity (see also [26]). Consequently and naturally in the equilibrium diffusion regime, the chemical potential of $\nu_{e}$ nearly vanishes in these regions. The authors of [35] speculated that the appearance of light nuclei (among them $\alpha$ particles as the dominant species) is causal for the development of such instability conditions. Just as Delfan Azari et al. [35], Abbar et al. [36] diagnosed ELN crossings in deep regions inside the PNS only in a small number of isolated points at the analyzed postbounce moments. They also correlated their occurrence with locations where the chemical potential of electron neutrinos nearly vanishes and pointed out that the electron fraction $Y_{e}$ is relatively low there and the temperature is close to maximal values.

These interesting results motivate the need to extend the search for fast instabilities to other state-of-the-art and fully self-consistent multidimensional SN models. However, most multi-D SN simulations [30,37-46] evolve only the (energy-dependent) angular integrals ("moments") of the neutrino phase-space distributions with time, and not the fully angle-dependent distributions. Reference [39], used in [32,35], is a welcome exception. The lack of detailed angular information seems to preclude a linear stability analysis that requires knowing these distributions. To overcome this limitation, some of us have recently proposed an alternative method to diagnose the possibility of fast instabilities in the absence of detailed knowledge of the ELN distributions [47]. This recipe is based on identifying a specific Fourier mode of the flavor instability field called the "zero mode," which has an easily calculable growth rate depending only on the angular moments of the ELN up to second order. It has been shown with numerical examples that the growth rate of this mode, calculated from 
the stability analysis, nicely approximates the growth of flavor conversions for the same mode in detailed numerical calculations.

The purpose of the present work is to use this new method of analysis to scan the different regions in selfconsistent, state-of-the-art 3D SN models with fully 3D two-moment neutrino transport for the possibility of fast flavor conversions therein. Specifically, we employ two time-dependent stellar core-collapse (and explosion) simulations for $9 M_{\odot}$ and $20 M_{\odot}$ progenitors recently published by the Garching group $[45,46]$. Both of these simulations exhibit the LESA phenomenon. We will demonstrate that the direct evaluation of discretized numerical data provided by computational models leads to the identification of only a few, isolated points of ELN crossings. We will argue that instead of being such pointlike locations, the regions of fast flavor instability are thin 2D layers that first appear around small 3D volumes in the convective layer of the PNS, and which grow with time as the convective and diffusive transport of the electron-lepton number drives a decrease of $Y_{e}$ in the PNS convection layer. We also observe a strong hemispheric asymmetry of the thin layer of flavor instability correlated with the asymmetry of PNS convection leading to the LESA phenomenon. The regions of ELN crossings are much more extended in the hemisphere opposite to the LESA dipole direction, where PNS convection is weaker and $Y_{e}$ is lower. Our analysis thus shows that the locations of ELN crossings are not dotlike and fluctuating because of stochastic hydrodynamical variations, but they are long-lasting and large-scale structures (for possibly important implications of this fact, see [34]).

In Sec. II, we describe our method for diagnosing instabilities based on the angular moments of the neutrino phase-space distributions. In Sec. III we present the results of our search for fast flavor instability in the two investigated 3D SN models, first by directly using the discretized output of the numerical simulations in the instability condition (Sec. III A), which leads to the identification of only a few isolated points of flavor instability located interior to the neutrinospheres in the convective shell of the PNS. In Sec. III B we discuss the conditions for ELN crossings. We argue that this direct analysis on the discrete numerical mesh fails to correctly identify the regions of flavor instability, which are actually thin 2D layers in 3D space. We propose an alternative, better strategy to find these layers containing the locations of flavor instability. In Sec. IV we present the time evolution of the instability layers in our two model runs (Sec. IVA) and explain the reason for the development of the relevant physical conditions (Sec. IV B). We also discuss their correlation with the dipole of the lepton-number emission and the asymmetry of the electron distribution in the PNS convection layer connected with the LESA phenomenon. Finally, in Sec. V, we conclude with a brief summary.

\section{FAST INSTABILITIES AND MOMENTS OF NEUTRINO DISTRIBUTIONS}

\section{A. Instability equation}

To track the existence of the fast neutrino flavor instability one has to perform a linear stability analysis of the neutrino equations of motion. We refer the interested reader to [22] for a detailed discussion of this analysis and for the related publications. Here, we simply mention that in the growing literature about fast flavor conversions, a novel approach to study these effects was recently proposed in [17]. This is based on the dispersion relation for the frequency and wave number $(\omega, \mathbf{k})$ in the mean field of $\nu_{e} \nu_{x}$ coherence, which is essentially the off-diagonal element of the neutrino density matrix $Q(\mathbf{p}, \mathbf{x}, t)$ that we will call $S$ in the following. One looks for solutions of the linearized equations for the flavor evolution in the form

$$
S \sim e^{i(\mathbf{k} \cdot \mathbf{x}-\omega t)}
$$

Typically such a solution may exist only if $\omega$ and $\mathbf{k}$ are related by an appropriate equation, called the dispersion relation. Loosely speaking, if either $\mathbf{k}$ or $\omega$ develop imaginary parts leading to positive real arguments in the exponential, the solution is expected to grow in space or time, thus signaling an "instability." Unfortunately, identifying the instabilities rests upon a more complicated analysis that requires a full characterization of the complex analytic structure of the dispersion relation [18].

In [47] some of us proposed a simpler analytical tool to diagnose the fast neutrino instability. Our proposal is based on identifying a specific Fourier mode of the flavor instability field that we call the "zero mode" in a corotating frame, in which one can gauge away the matter term from the neutrino equations of motion. We labeled this zero mode as $\mathbf{k}=0{ }^{1}$

This is motivated by the fact that the calculation of $\omega$ for this mode is significantly simpler than a full characterization of the dispersion relations, $D(\omega, \mathbf{k})$ [18]. In fact, for this mode the dispersion relation becomes

$$
D(\omega, 0)=\operatorname{det}\left(\eta^{\mu \nu}+\frac{1}{\omega} V^{\mu \nu}\right)=0
$$

with $\eta^{\mu \nu}=\operatorname{diag}(+1,-1,-1,-1)$, i.e., $D(\omega, 0)$ is a polynomial in $\omega$. The specific model of SN neutrino populations and their angular distributions, encoded in the ELN, only enters the equation through the tensor $V^{\mu \nu}$

\footnotetext{
${ }^{1}$ A remark is in order. Our method does not completely exclude the presence of an instability for those points where we find $\operatorname{Im}(\omega)=0$. Indeed, as our analysis is based only on the zero mode $\mathbf{k}=0$, there might be a $\mathbf{k} \neq 0$ that is unstable. Therefore, one cannot exclude larger instability regions than those that we will show. The reader is referred to [48] for a comparison of instability regions obtained with different instability criteria.
} 
(with $\mu, \nu=0,1,2,3$ ) that contains the angular moments of the neutrino distributions up to second order in the neutrino velocity, namely,

$$
V^{\mu \nu}=\int \frac{d \mathbf{v}}{4 \pi} v^{\mu} v^{\nu} G_{\mathbf{v}},
$$

where $v^{\mu}=(1, \mathbf{p} / E)$, i.e., the zeroth component of the velocity four-vector is 1 and the spatial components are given by the unit vector $\mathbf{v}=\mathbf{p} / E$ (with $E=|\mathbf{p}|$ ). The function

$$
G_{\mathbf{v}}=\sqrt{2} G_{F} \int_{0}^{\infty} \frac{d E E^{2}}{2 \pi^{2}}\left[f_{\nu_{e}}(E, \mathbf{v})-f_{\bar{\nu}_{e}}(E, \mathbf{v})\right]
$$

is the difference of the phase-space occupation functions integrated over energy space, i.e., the angular distribution of the ELN [15]. Here we assume that $\nu_{x}$ and $\bar{\nu}_{x}$ have identical distributions. Therefore, $V^{\mu \nu}$ depends on the angular moments of the neutrino-species dependent phase space distributions up to second order in the neutrino velocities, i.e.,

$$
V^{\mu \nu}=\left\langle v^{\mu} v^{\nu}\right\rangle_{\nu_{e}}-\left\langle v^{\mu} v^{\nu}\right\rangle_{\bar{\nu}_{e}},
$$

where the notation $\langle\cdots\rangle_{\nu_{\alpha}}$ refers to

$$
\langle\cdots\rangle_{\nu_{\alpha}} \equiv \sqrt{2} G_{F} \int \frac{d^{3} \mathbf{p}}{(2 \pi)^{3}}(\cdots) f_{\nu_{\alpha}}(\mathbf{p})
$$

These (energy-integrated) moments are related to the difference of the number densities $\left(n_{\nu}\right)$ of $\nu_{e}$ and $\bar{\nu}_{e}$, the corresponding difference of the number-flux densities $\left(F_{\nu}^{r}\right)$, and the difference of the second angular moments of the $\nu_{e}$ and $\bar{\nu}_{e}$ number distributions $\left(P_{\nu}^{r r}\right)$ as follows:

$$
\begin{gathered}
V^{00}\left(\sqrt{2} G_{F}\right)^{-1}=n_{\nu_{e}}-n_{\bar{\nu}_{e}} \equiv \Delta n_{\nu}, \\
V^{0 r}\left(\sqrt{2} G_{F}\right)^{-1}=\left(F_{\nu_{e}}^{r}-F_{\bar{\nu}_{e}}^{r}\right) c^{-1} \equiv \Delta F_{\nu} c^{-1}, \\
V^{r r}\left(\sqrt{2} G_{F}\right)^{-1}=P_{\nu_{e}}^{r r}-P_{\bar{\nu}_{e}}^{r r} \equiv \Delta P_{\nu}^{r r},
\end{gathered}
$$

where for later quantitative evaluation we have reintroduced the factors $c$ in the expressions in the right-hand side (RHS) of these relations.

We remind the reader that the two-moment "M1" neutrino-transport scheme used in $[45,46]$ evolves the " 00 " and the " $0 i$ " components (with $i \in\{r, \theta, \phi\}$ being radius $r$, polar angle $\theta$, and azimuthal angle $\phi$ of the polar coordinate system) of the moments in Eq. (6) in time for all neutrino species. The system of moment equations of the transport solver is closed by an algebraic relation for nonevolved moments (i.e., for the " $i j$ " components of the tensor with $i \geq 1$ and $j \geq 1$ ), which depend on the " 00 " and the " $0 i$ " components (see, e.g., [49]).

\section{SEARCH FOR FAST INSTABILITIES}

\section{A. Direct analysis of discretized numerical results}

In this work we employ energy-integrated angular moments of the neutrino number distribution provided by the neutrino transport solver used in the considered 3D SN simulations of Refs. [45,46], Models s9.0 FMD H and s20 FMD $H$ there. Appropriate normalization constants as specified in Eqs. (7)-(9) are applied in order to match the quantities in Eq. (3). We choose to work mainly in the comoving frame of the stellar fluid (fluid frame), where SN neutrino transport usually provides its output quantities. Equivalent results in terms of flavor instabilities are obtained in the laboratory frame, i.e., the rest frame of the stellar center. We will briefly demonstrate this later.

We mainly focus on our progenitor with $9 M_{\odot}$ and discuss similarities as well as differences compared to the $20 M_{\odot}$ simulation. Figure 1 displays Aitoff projections of the $9 M_{\odot}$ model for postbounce times of $t=300 \mathrm{~ms}$ and $500 \mathrm{~ms}$ (upper two panels) and of the $20 M_{\odot}$ star for $t=$ $200 \mathrm{~ms}$ after bounce (bottom panel). Different radial directions in the 3D simulations correspond to discretized values of the zenith angle $\theta$ and azimuthal angle $\phi$. Points $(\phi, \theta)$ are marked by green color if the solutions of Eq. (2) yield $\operatorname{Im}(\omega)>0$ for at least one value of the discretized radial coordinate $r$ in the range $10 \mathrm{~km} \leq r \leq 30 \mathrm{~km}$. If the color is blue, then $\operatorname{Im}(\omega)=0$ for all radii; i.e., there is no instability.

In the $9 M_{\odot}$ model at $300 \mathrm{~ms}$ we find instability points only in the interval of $[12,15] \mathrm{km}$ and for very specific directions, i.e., a few isolated and unconnected pairs of values $(\phi, \theta)$. As specified in the plots, $\operatorname{Im}(\omega) \sim$ $O(10-100) \mathrm{m}^{-1}$ at unstable locations, which means the flavor instability can develop over a timescale even shorter than a nanosecond. In the middle and lower panels of Fig. 1 we witness a larger number of points of instability than in the upper panel.

Nonzero values of $\operatorname{Im}(\omega)$ in the $9 M_{\odot}$ model occur only later than $\sim 300 \mathrm{~ms}$ after bounce, whereas in the $20 M_{\odot}$ the condition for flavor instability shows up earlier and more points with a positive imaginary part of $\omega$ are present already at $200 \mathrm{~ms}$.

We note in passing that we have not detected any points of flavor instability between the neutrino-decoupling region and the SN shock (at $r \geq 30-50 \mathrm{~km}$, depending on the postbounce time). Some authors have speculated about the possibility that ELN crossings might occur in this region in multidimensional models and, indeed, Ref. [31] as well as Refs. $[36,50]$ have reported such locations in $2 \mathrm{D}$ and 3D simulations of an $11.2 M_{\odot}$ star. In this context, however, it is important to keep in mind that our moment-based criterion employs differences of angle and energy integrals of the neutrino distribution functions. Such an integral criterion is not necessarily sensitive enough to diagnose low-level crossings in the angular distributions of $\nu_{e}$ and $\bar{\nu}_{e}$, 


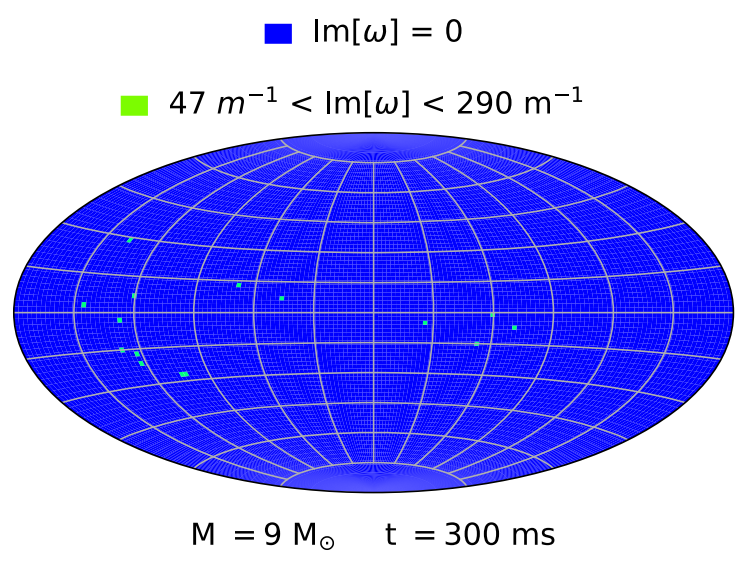

$\mathrm{R} \in[12,15] \mathrm{km}$

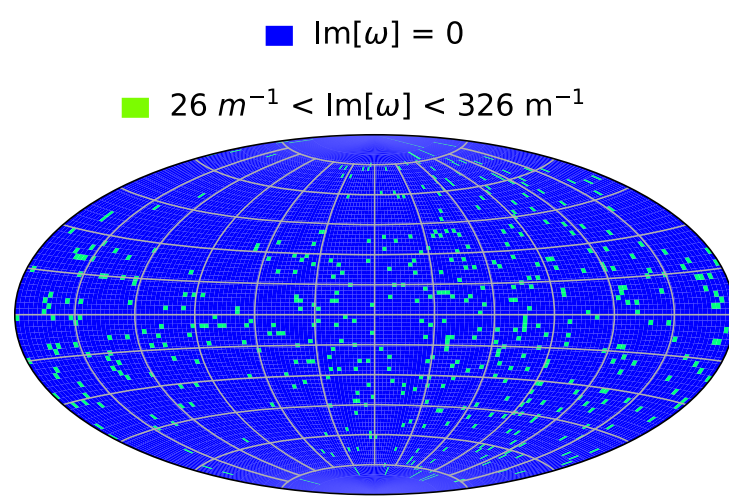

$M=9 M_{\odot} \quad t=500 \mathrm{~ms}$

$R \in[10,16] \mathrm{km}$

$\operatorname{Im}[\omega]=0$

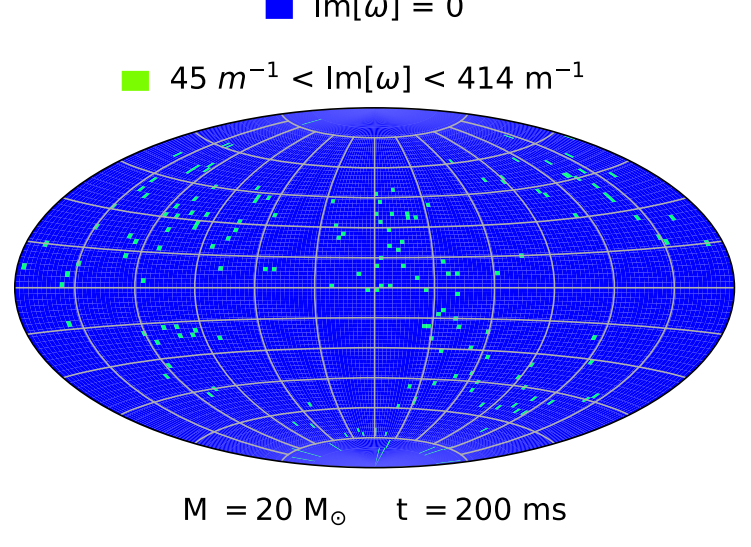

$R \in[11,16] \mathrm{km}$

FIG. 1. Aitoff projections for the $\log _{10} \operatorname{Im}[\omega]$ obtained by solving Eq. (2) in the case of the $9 M_{\odot}$ star at $t=300 \mathrm{~ms}$ and $500 \mathrm{~ms}$ after core bounce (upper two panels) and of the $20 M_{\odot}$ star at $200 \mathrm{~ms}$ post bounce (bottom panel). The plots are obtained by selecting the largest $\log _{10} \operatorname{Im}[\omega]$ in the radial range of $[10,30] \mathrm{km}$. i.e., small differences in the distribution functions leading to a reversed ordering of the distributions in some narrow region or in a sparsely populated part of the angle space, as, e.g., spotted in the preshock domain as a consequence of a small fraction of neutrinos that are backscattered in collisions with infalling nuclei [33].

Our findings are reminiscent of the individual points of ELN crossings that were identified deep inside the PNS by [35] in the 2D simulation of this $11.2 M_{\odot}$ model and by [36] in the 3D simulations of the same $11.2 M_{\odot}$ progenitor and of a $27 M_{\odot}$ model. In the following section we will argue that the regions of fast-flavor instability form thin $2 \mathrm{D}$ layers in 3D space rather than isolated points, and the identification of individual points in our analysis is an artifact connected with the discretization of the physical variables in the numerical treatment. This conclusion does probably also apply to the results of the previous investigations in Refs. [35,36].

\section{B. Conditions for ELN crossings}

Similar to Refs. [35,36] our points of flavor instability are located deep inside the PNS, i.e., below the neutrinospheres in a region where neutrinos diffuse and their phasespace distributions are very close to those of local chemical equilibrium.

Before we discuss in detail why we-and probably also the references mentioned above-have found only isolated points instead of extended regions of flavor instability, we will introduce a simplified and approximative criterion for the instability, which makes the underlying physics more transparent. To achieve this, we take advantage of the fact that the nonradial moments are much smaller than the radial ones in the diffusive core where we find instability. Moreover, in this region $V^{r r}=V^{\theta \theta}=V^{\phi \phi}$ holds because the neutrino phase-space distributions are nearly isotropic. In this limit, which effectively corresponds to the 1D case, Eq. (2) is explicitly quadratic in $\omega$, namely

$$
\left(\omega+V^{00}\right)\left(\omega-V^{r r}\right)+\left(V^{0 r}\right)^{2}=0,
$$

with the solution

$\omega=\frac{1}{2}\left(V^{r r}-V^{00} \pm \sqrt{\left(V^{00}+V^{r r}\right)^{2}-4\left(V^{0 r}\right)^{2}}\right)$.

With the additional relation that $V^{r r}=\frac{1}{3} V^{00}$, which is valid to high accuracy in the diffusion regime, the condition for instability becomes

$$
\mathcal{F}=\frac{16}{9}\left(V^{00}\right)^{2}-4\left(V^{0 r}\right)^{2}<0 .
$$

In terms of the differences of number densities $\Delta n_{\nu}$ and radial fluxes $\Delta F_{\nu}^{r}$ of $\nu_{e}$ and $\bar{\nu}_{e}$ [see Eqs. (7) and (8)] this instability condition reads 


$$
\mathcal{F}\left(\sqrt{2} G_{F}\right)^{-1}=\frac{16}{9}\left(\Delta n_{\nu}\right)^{2}-\frac{4}{c^{2}}\left(\Delta F_{\nu}^{r}\right)^{2}<0 .
$$

The presence of flavor instability is thus mostly dependent on $V^{00}$ and $V^{0 r}$, i.e., on the differences of the neutrino number densities and number-flux densities of $\nu_{e}$ and $\bar{\nu}_{e}$. We explicitly checked that Eq. (11) gives almost identical results to what is shown in Fig. 1.

A subtlety concerns the evaluation of the instability condition either with the moments in the reference frame comoving with the stellar fluid, where the neutrino quantities (i.e., the angular moments) are computed by the numerical transport code, or in the laboratory frame. In a full-angle treatment of the neutrino transport this corresponds to the question whether ELN crossings shall be searched for with the angular distributions of $\nu_{e}$ and $\bar{\nu}_{e}$ in the comoving frame or in the lab frame. We will show here that at the level of the angular moments, which we use for our analysis, the results are basically independent of the specific frame where the neutrino moments are evaluated. For fluid velocities $\tilde{v} \ll c$, the lab-frame and comoving frame moments are related to the lowest order in $\tilde{v} / c$ through

$$
\begin{gathered}
n_{\nu}^{\mathrm{lab}} \approx n_{\nu}, \\
F_{\nu}^{r, \mathrm{lab}} \approx F_{\nu}^{r}+\tilde{v}_{r} n_{\nu}, \\
P_{\nu}^{r r, \mathrm{lab}} \approx P_{\nu}^{r r} .
\end{gathered}
$$

In the frame transformations of $n_{\nu}$ [Eq. (14)] and $P_{\nu}^{r r}$ [Eq. (16)] we omit terms such as $\frac{1}{c^{2}} \tilde{v}_{i} F_{\nu}^{i}$ and $\frac{1}{c^{2}} \tilde{v}^{r} F_{\nu}^{r}$, respectively. Since $\tilde{v} \ll c$ holds and in the diffusion regime interior to the neutrinospheres also $\frac{1}{c}\left|F_{\nu}^{i}\right| \ll n_{\nu}$ applies, ${ }^{2}$ the disregarded terms are many orders of magnitude smaller than the leading ones that we retain in Eqs. (14) and (16). Replacing the comoving-frame quantities in Eq. (13) by the lab-frame ones of Eqs. (14)-(16), we obtain

$$
\begin{aligned}
\mathcal{F}\left(\sqrt{2} G_{F}\right)^{-1}= & \frac{16}{9}\left(\Delta n_{\nu}^{\mathrm{lab}}\right)^{2}\left(1-\frac{9}{4} \frac{\tilde{v}_{r}^{2}}{c^{2}}\right) \\
& -\frac{4}{c^{2}} \Delta F_{\nu}^{r, \mathrm{lab}}\left(\Delta F_{\nu}^{r, \mathrm{lab}}-2 \tilde{v}_{r} \Delta n_{\nu}^{\mathrm{lab}}\right) \\
< & 0 .
\end{aligned}
$$

Since $\tilde{v}_{r} \ll c$ and, as we shall argue below, the condition can be fulfilled only when $\Delta n_{\nu} \approx \Delta n_{\nu}^{\text {lab }} \approx 0$, the relation in Eq. (17) is basically identical with

\footnotetext{
${ }^{2}$ This can easily be verified by comparing the left panels in the top and third rows of Fig. 2 for $r \lesssim 30 \mathrm{~km}$ and taking into account that $\left|F_{\nu}^{\theta}\right| \approx\left|F_{\nu}^{\phi}\right| \lesssim\left|F_{\nu}^{r}\right|$.
}

$$
\mathcal{F}\left(\sqrt{2} G_{F}\right)^{-1} \approx \frac{16}{9}\left(\Delta n_{\nu}^{\text {lab }}\right)^{2}-\frac{4}{c^{2}}\left(\Delta F_{\nu}^{r \text { lab }}\right)^{2}<0,
$$

which is identical to the instability condition of Eq. (13).

Figure 2 confirms that indeed it does not matter whether the analysis is performed with lab-frame or comovingframe moments for the neutrinos. The figure shows, in both reference frames, radial profiles of the number densities $n_{\nu}$ of $\nu_{e}$ and $\bar{\nu}_{e}$ individually and their difference for the $9 M_{\odot}$ model at a postbounce time of $300 \mathrm{~ms}$ (four upper left panels); the corresponding second angular moments $P_{\nu}^{r r}$ and their difference (four upper right panels); the radial neutrino-flux densities $F_{\nu}^{r}$ and their difference (four lower left panels); and the "flavor-instability functional" $\mathcal{F}$ of Eqs. (13) and (18) (four lower right panels). The angular direction $(\theta, \phi)$ for the radial ray was chosen such that one of the instability points visible in the top plot of Fig. 1 was crossed. This can be seen in the four panels on the lower right of Fig. 2, where at $r \approx 14 \mathrm{~km}$ the flavor-instability condition is fulfilled. The four upper left panels demonstrate that at this location $n_{\nu_{e}}$ and $n_{\bar{\nu}_{e}}$ are approximately equal. Lab-frame and comoving-frame quantities exhibit exactly the same behavior.

A comparison of the four upper left and four upper right panels shows that the same conclusion can be drawn from inspection of $P_{\nu}^{r r}$, because in the diffusion region $P_{\nu}^{r r}=\frac{1}{3} n_{\nu}$ is very well fulfilled. This relation does not hold any longer when neutrinos begin to decouple from the stellar medium near the neutrinosphere and undergo the transition to free streaming outside. In this case $P_{\nu}^{r r} \rightarrow n_{\nu}$ asymptotically for $r \rightarrow \infty$, and therefore our flavor-instability conditions of Eqs. (13) and (18) are not valid any more. In the displayed model this is the case for radii $r \gtrsim 30 \mathrm{~km}$, for which reason the negative values of the flavor-instability functional for $r \gtrsim 40 \mathrm{~km}$ do not signal flavor instability in this region exterior to the PNS.

The two lower right bottom panels of Fig. 2 also display the term $\frac{16}{9}\left(\Delta n_{\nu}\right)^{2}$ as part of the flavor-instability functional for comparison with the full expression. One can see that this term usually dominates the second one, $\frac{4}{c^{2}}\left(\Delta F_{\nu}^{r}\right)^{2}$, by several (typically by $2-3$ ) orders of magnitude. This can also be directly verified by comparing $\Delta n_{\nu}$ in the upper left panels with $\frac{1}{c} \Delta F_{\nu}^{r}$ displayed in the lower left panels. We remark in passing that strongly negative values of the $\bar{\nu}_{e}$ flux in the comoving frame occur because of a local temperature maximum that drives the diffusion flux of $\bar{\nu}_{e}$ inward while the more degeneracy-driven diffusion flux of $\nu_{e}$ can still be outward directed. Although the lab-frame and comoving-frame fluxes are considerably different (because the advective component $v_{r} n_{\nu}$ can dominate the diffusive component in the convection layer of the PNS), the radial profiles of $\Delta F_{\nu}^{r}$ are more similar for lab-frame and comoving-frame fluxes, and the instability functional $\mathcal{F}$ in the lower right panels does not exhibit any visible frame dependence. 

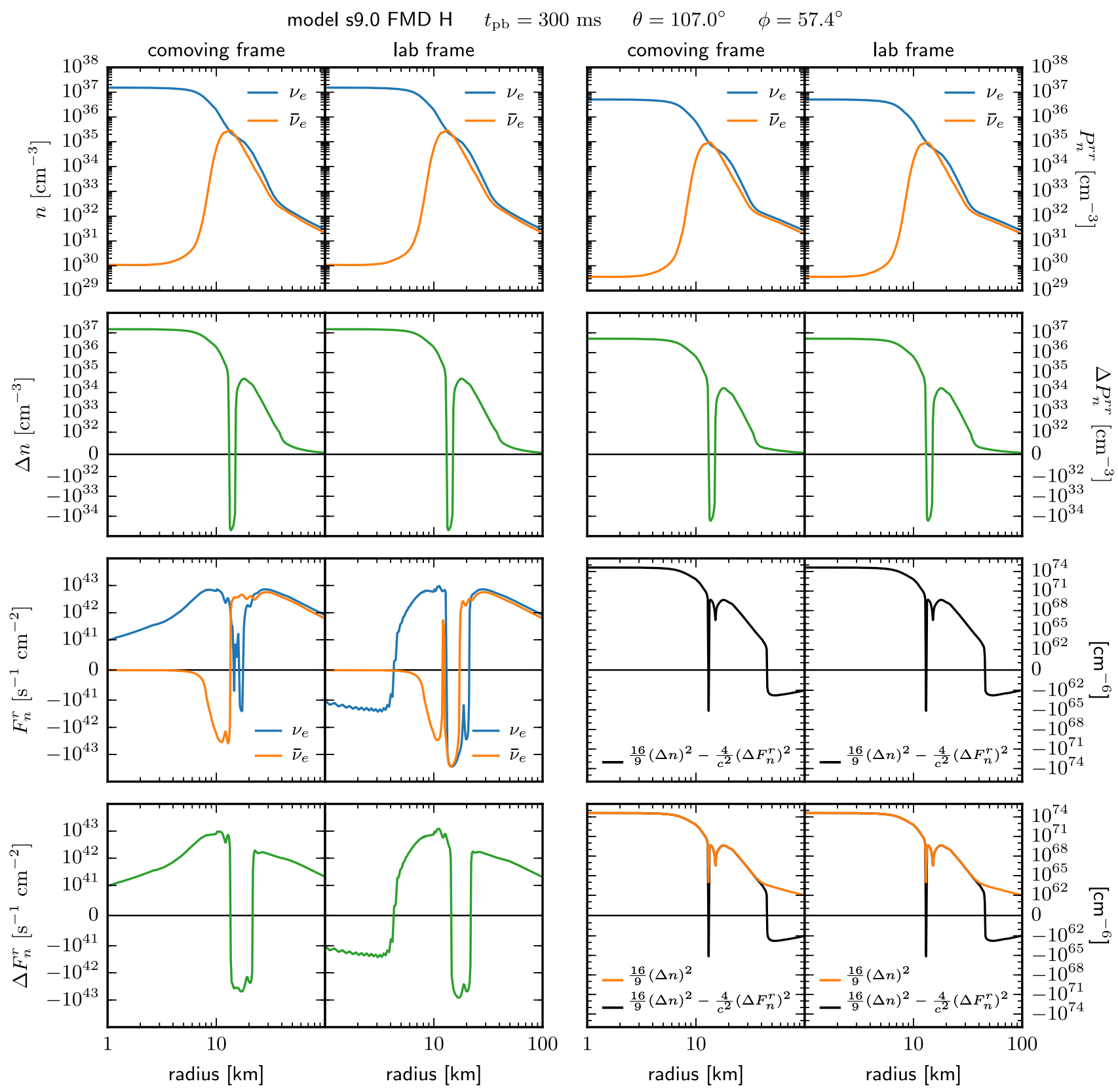

FIG. 2. Radial profiles of the basic (energy-integrated) angular moments $n_{\nu}, F_{\nu}^{r}$, and $P_{\nu}^{r r}$ for $\nu_{e}$ and $\bar{\nu}_{e}$ as well as their differences $\Delta n_{\nu}$, $\Delta F_{\nu}^{r}$, and $\Delta P_{\nu}^{r r}$ along a radial direction at $(\theta, \phi)=\left(107^{\circ}, 57.4^{\circ}\right)$ in our $9 M_{\odot}$ model at $300 \mathrm{~ms}$ after bounce. The four panels on the lower right display the corresponding flavor-instability functional $\mathcal{F}$ and, for comparison, the first term of it, $\frac{16}{9}\left(\Delta n_{\nu}\right)^{2}$, as labeled in the panels.

There are severe consequences of this huge imbalance between the first and the second terms in the flavorinstability functional $\mathcal{F}$ when searching for ELN crossing points by evaluating the functional with discretized numerical results. To detect such points, i.e., in order to find grid locations where $\mathcal{F}<0$, the term $\frac{16}{9}\left(\Delta n_{\nu}\right)^{2}$ must be very close to zero at exactly such grid positions, because only then can the small second term lead to a negative value of $\mathcal{F}$. If, however, the discrete grid points are too far away from the root of $\mathcal{F}$, the values of $\frac{16}{9}\left(\Delta n_{\nu}\right)^{2}$ at these points may be so large that the second term $\frac{4}{c^{2}}\left(\Delta F_{\nu}^{r}\right)^{2}$ does not achieve to produce negative values of $\mathcal{F}$. This, in fact, is likely to happen in the far majority of all cases where the physical conditions enable flavor instability, and only in a minor fraction of such locations do the discretized spatial points of the computational grid coincide incidentally with locations where the combination of terms can yield $\mathcal{F}<0$, and thus signal the presence of flavor-unstable conditions.

An example of such a missed point of instability can also be spotted in Fig. 2. The plots of $\mathcal{F}$ in the lower right panels 

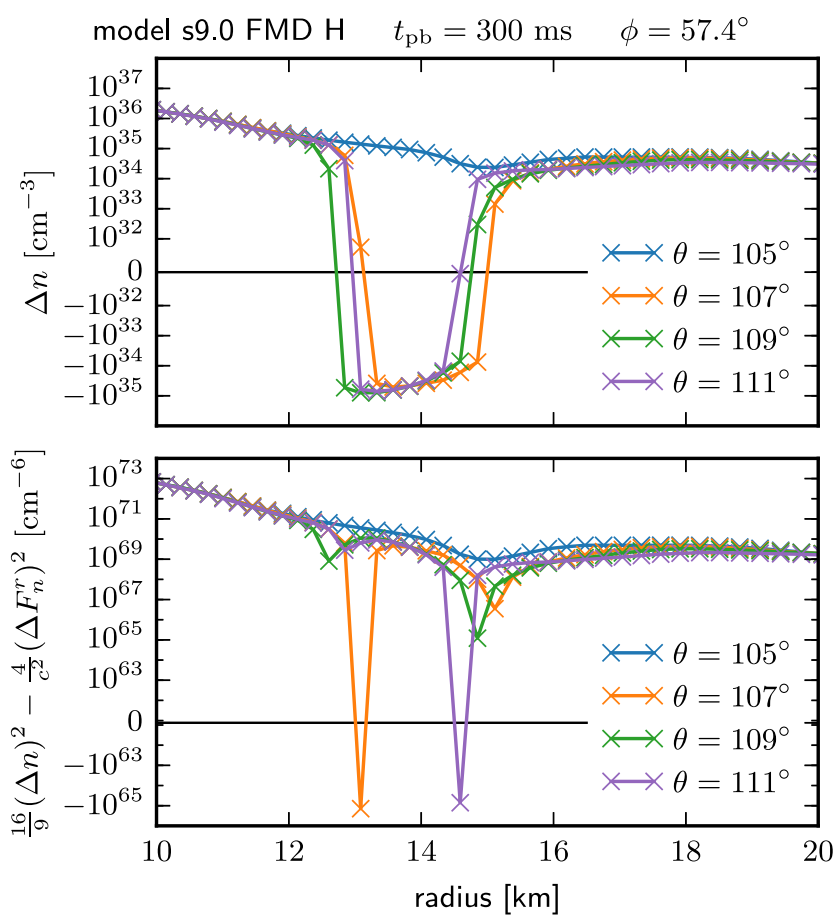

FIG. 3. Radial profiles of $\Delta n_{\nu}$ (upper panel) and $\mathcal{F}$ (lower panel) in the vicinity of the two local minima of $\mathcal{F}$ visible around $\sim 14 \mathrm{~km}$ in Fig. 2 in the case of our $9 M_{\odot}$ simulation at $300 \mathrm{~ms}$ after bounce. Besides the radial direction chosen for Fig. 2, three other radial directions with neighboring zenith angles for a fixed azimuthal angle of $\phi=57.4^{\circ}$ are shown, too. The individual radial mesh points of the computational grid are marked by crosses.

show two local minima between $10 \mathrm{~km}$ and $20 \mathrm{~km}$. Only in the case of the left one does the minimum of $\mathcal{F}$ reach a negative value, but for the right one the minimum is still on the positive side. Is the condition of flavor instability also fulfilled at this position and just not detected by the numerical analysis? Indeed, this is the situation as visualized in detail by a close-up of the region of the two local minima of $\mathcal{F}$ in Fig. 3 with crosses marking the positions of all radial mesh points of the computational grid. The orange line in the two panels corresponds to the radial direction chosen for the profiles in Fig. 2. It is obvious that $\Delta n_{\nu}$ (upper panel of Fig. 3) has two zero crossings and becomes negative between these two roots. If a mesh point happens to be close to the root, the small, second term in Eqs. (13) and (18) achieves to drive $\mathcal{F}$ (lower panel) to the negative side. Such a situation occurs for the left one of the two roots along the radial direction at $(\theta, \phi)=\left(107^{\circ}, 57.4^{\circ}\right)$ and for the right root in the case of $(\theta, \phi)=\left(111^{\circ}, 57.4^{\circ}\right)$. If, however, the mesh points are too far away from the root, then $\mathcal{F}$ remains positive at all discrete points of the grid. This is the situation for the direction corresponding to $(\theta, \phi)=\left(109^{\circ}, 57.4^{\circ}\right)$ displayed in Fig. 3, although also in this case $\Delta n_{\nu}$ possesses two roots. The fourth selected case in this figure for $(\theta, \phi)=\left(105^{\circ}, 57.4^{\circ}\right)$ does not exhibit any change in the sign of $\Delta n_{\nu}$.
Discretization effects are therefore the reason why only very few points with ELN crossings could be identified by the analysis so far. Consequently, only individual, isolated points of instability appeared on the three panels of Fig. 1. This problem would become even more severe if the resolution of the computational grid used in our SN simulations had been coarser.

Our case is only an example how numerical discretization effects may impede the possibility to detect flavorunstable conditions that occur in narrowly delimited spatial regions. A specific condition, such as, e.g., our instability criteria of Eqs. (2), (13), and (18) or any other analytical criterion relating physical quantities that are available on a discrete numerical mesh, may fail to identify the spatial locations of instability. We speculate that also the investigations in Refs. [35,36], determining ELN crossings by using angular distributions, suffered from the finite numerical resolution of the underlying SN simulations and therefore failed to find more spatial points of flavor-unstable conditions.

Actually, the problems encountered in our analysis with discretized physical variables can easily be circumvented. Evaluating the relations of Eqs. (13) or (18) to search for spatial locations where $\mathcal{F}<0$ (or for roots of $\mathcal{F}$ ) on a mesh of discrete points is not a promising strategy. Instead, it is preferable to look for regions where $\Delta n_{\nu}$ changes its sign, which we understood as a sufficient condition to obtain roots of $\mathcal{F}{ }^{3}$ When $\Delta n_{\nu}$ changes its sign between two grid points, there must be a root of this quantity between the two points. Close to this root $\mathcal{F}$ will become negative, unless $\Delta F_{\nu}^{r}$ vanishes in this region. In such a pathological and very rare situation our approximate flavor-instability criterion based on a few angular moments of the neutrino phase-space distributions does not provide conclusive information.

\section{TIME EVOLUTION AND PHYSICAL CONDITIONS FOR ELN CROSSINGS}

\section{A. Time evolution}

With the approximative but numerically robust criterion of sign changes of $\Delta n_{\nu}$, we have evaluated our $9 M_{\odot}$ and $20 M_{\odot}$ models in time to track the evolution of the volume of ELN crossings interior to the PNS in our $9 M_{\odot}$ and $20 M_{\odot}$ simulations.

\footnotetext{
${ }^{3}$ Strictly speaking, a sign change of $\Delta n_{\nu}$ is not necessary to get $\mathcal{F}<0$, but this condition for $\mathcal{F}$ can also be fulfilled when $\Delta n_{\nu}$ dips nearly to zero while still remaining positive. However, because $\Delta n_{\nu}$ and $\frac{1}{c} \Delta F_{\nu}^{r}$ are orders of magnitude different in the diffusion regime (compare left panels in the second and fourth rows of Fig. 2), such a situation is highly fine-tuned and not as common in hot PNSs as sign changes of $\Delta n_{\nu}$. This is obvious from our analysis of the time evolution of PNSs in two 3D SN simulations.
} 

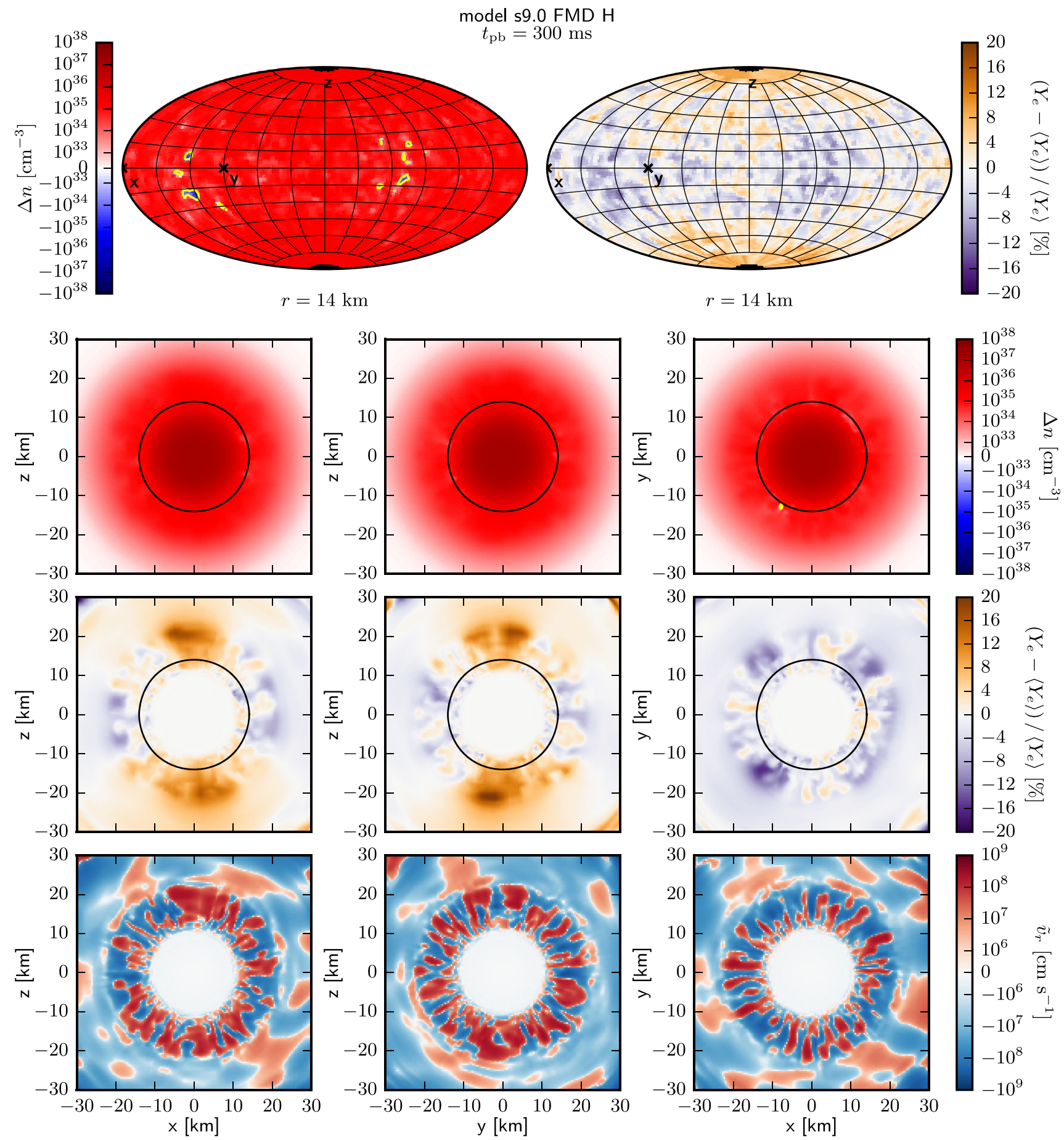

FIG. 4. Top row: Aitoff projections of $\Delta n_{\nu}=n_{\nu_{e}}-n_{\bar{\nu}_{e}}$ (left) and the variations of $Y_{e}$ relative to the angle-averaged value (right) at a radius of $14 \mathrm{~km}$ inside the PNS and postbounce time of $300 \mathrm{~ms}$ for our $9 M_{\odot}$ model. The directions of the $x, y$, and $z$ axes of the computational polar grid are denoted by black crosses. Second row: Corresponding cross-sectional cuts in the $x-z, y-z$, and $x-y$ planes with $\Delta n_{\nu}$ color coded. Third row: Variations of $Y_{e}$ in these cut planes. The radius of $r=14 \mathrm{~km}$ is marked by black circles. Bottom row: Radial velocities of the stellar plasma, $\tilde{v}_{r}$, in the cut planes. The convective shell in the PNS is visible by the quasiregular pattern of convection cells. In the plots of $\Delta n_{\nu}$ red indicates positive values, blue indicates negative values, the boundaries between both are locations with $\Delta n_{\nu} \approx 0$, where flavor instability, i.e., ELN crossings, is expected (highlighted by yellow lines). The first small raisinlike volumes with $\bar{\nu}_{e}$ excess signaled by negative $\Delta n_{\nu}$ can be found at locations of particularly low $Y_{e}$ (intense blue hues for negative $Y_{e}$ fluctuations relative to the average value). The spatial variations of $Y_{e}$ are connected to lepton-rich convective updrafts, which carry the electron-lepton number from the convectively stable PNS core outward, and more lepton-poor convective downdrafts. Larger red patches that mark buoyantly rising plasma in the bottom panels are therefore correlated with bigger orange regions in the panels of the third row, and more extended blue inward flows in the bottom panels coincide with the deepest-blue areas in the panels of the third row. 


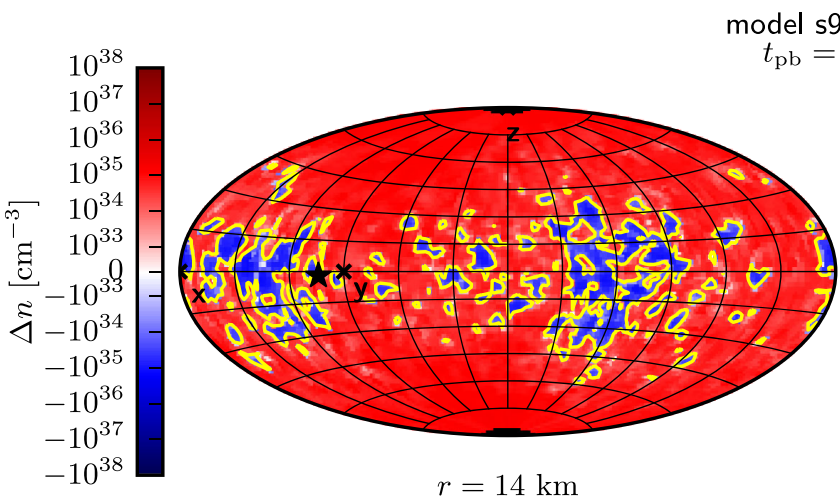

del s9.0 FMD H

b $=400 \mathrm{~ms}$
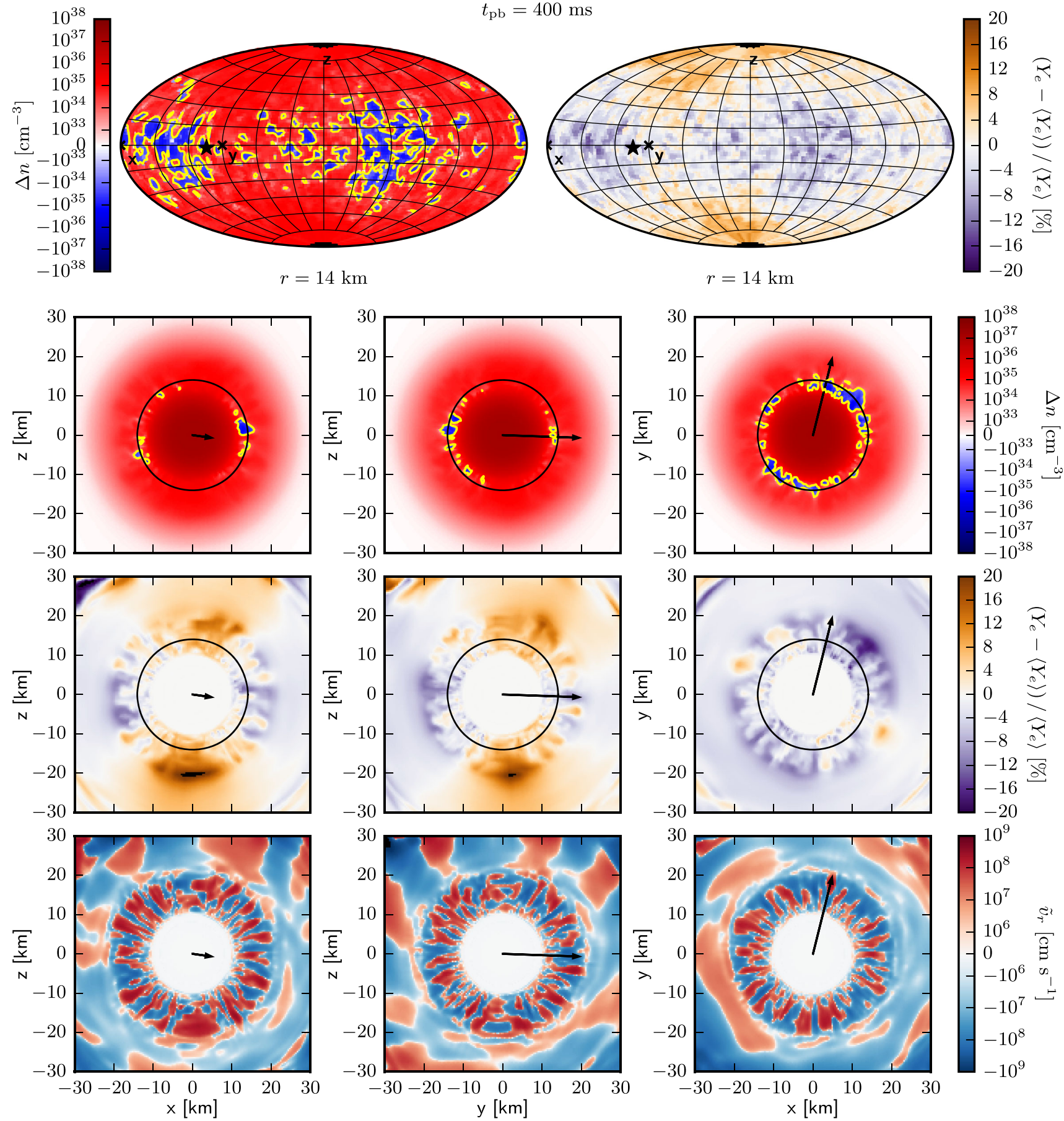

FIG. 5. Same as Fig. 4, but at $400 \mathrm{~ms}$ after core bounce. At this time the LESA lepton-emission dipole has become prominent and its direction is indicated by a black asterisk on the Aitoff projections and by a black arrow in the cross-sectional cuts. During all of the model evolution the LESA dipole vector direction is close to the $+y$ axis (see Model s9.0 FMD H in Fig. 3 of [45]).

For the $9 M_{\odot}$ model we show $\Delta n_{\nu}$ and the normalized fluctuations of the electron fraction, $\left(Y_{e}-\left\langle Y_{e}\right\rangle\right) /\left\langle Y_{e}\right\rangle$ (the angle brackets indicate averages over zenith and azimuthal angles), in full-sphere Aitoff projections at a radius of $r=14 \mathrm{~km}$ as well as cross-sectional cuts in the $x-z, y-z$, and $x-y$ planes at postbounce times of
300, 400, 500, and $600 \mathrm{~ms}$ in Figs. 4, 5, 6, and 7, respectively.

The fluctuations of $Y_{e}$ are connected to convective updrafts and downdrafts in the convection layer of the PNS (see Refs. [30,45]). Convective updrafts carry electron-lepton number from the convectively stable 


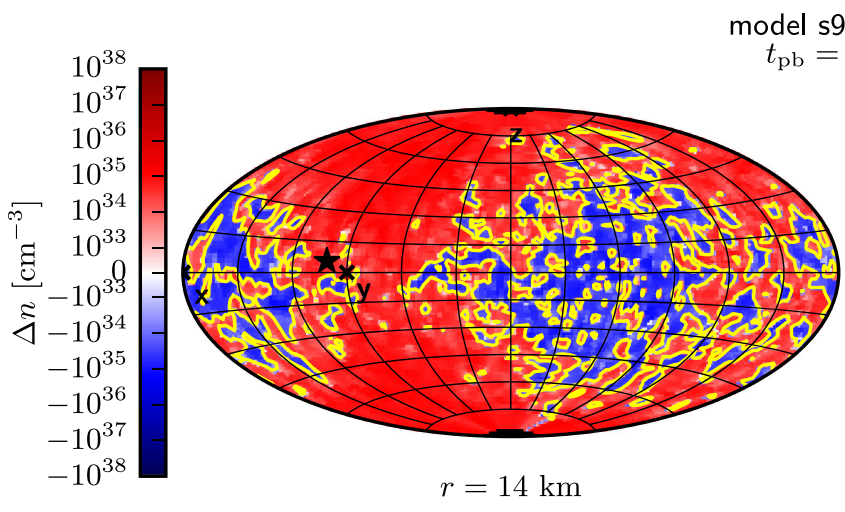

del s9.0 FMD H

$t_{\mathrm{pb}}=500 \mathrm{~ms}$
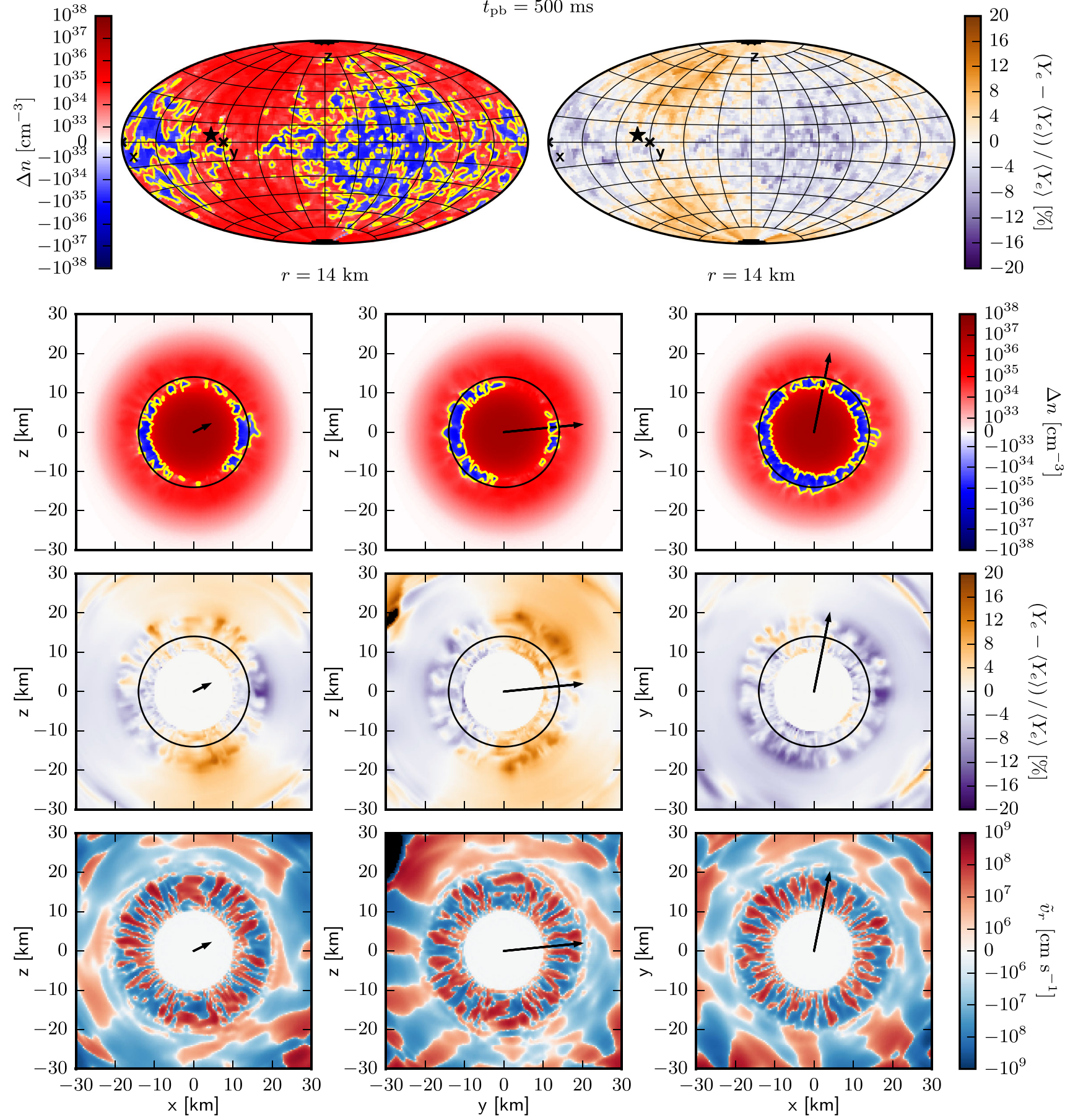

FIG. 6. Same as Fig. 4, but at $500 \mathrm{~ms}$ after core bounce. Regions of negative $\Delta n_{\nu}$ are more widespread in the anti-LESA direction where $Y_{e}$ is lower.

high-density core of the PNS outward and therefore exhibit higher values of $Y_{e}$ than the angle average. In contrast, convective downdrafts are more lepton poor, which also means that they contain more neutrons, which makes them specifically heavier so that they sink inward. The pattern of $Y_{e}$ fluctuations mirrors the familiar cell pattern of convection in spherical shells.
The zero crossings of $\Delta n_{\nu}$, and thus the locations very close to the flavor instability, are highlighted by yellow lines surrounding the volumes of negative values colored in blue. The physical thickness of these boundary layers of the $\Delta n_{\nu}<0$ volumes, i.e., the "skins" in which the flavor-instability condition is fulfilled, can be roughly estimated from Eqs. (13) or (18) by making 


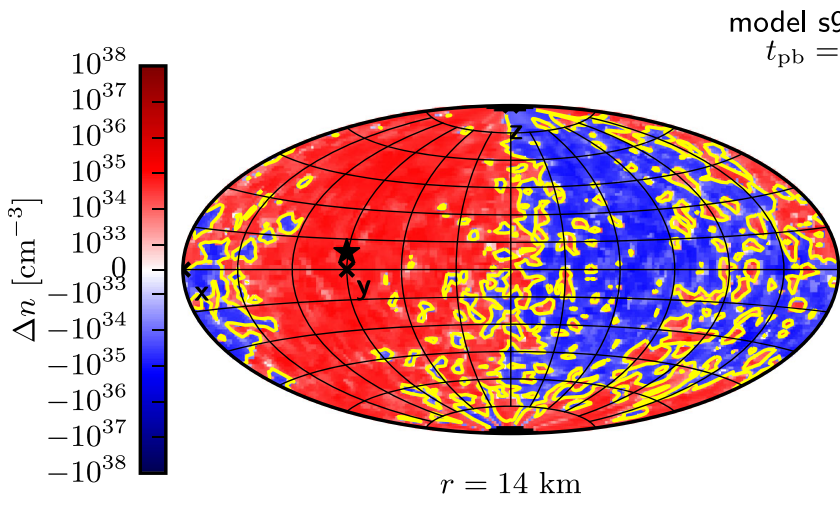

del s9.0 FMD H

$=600 \mathrm{~ms}$
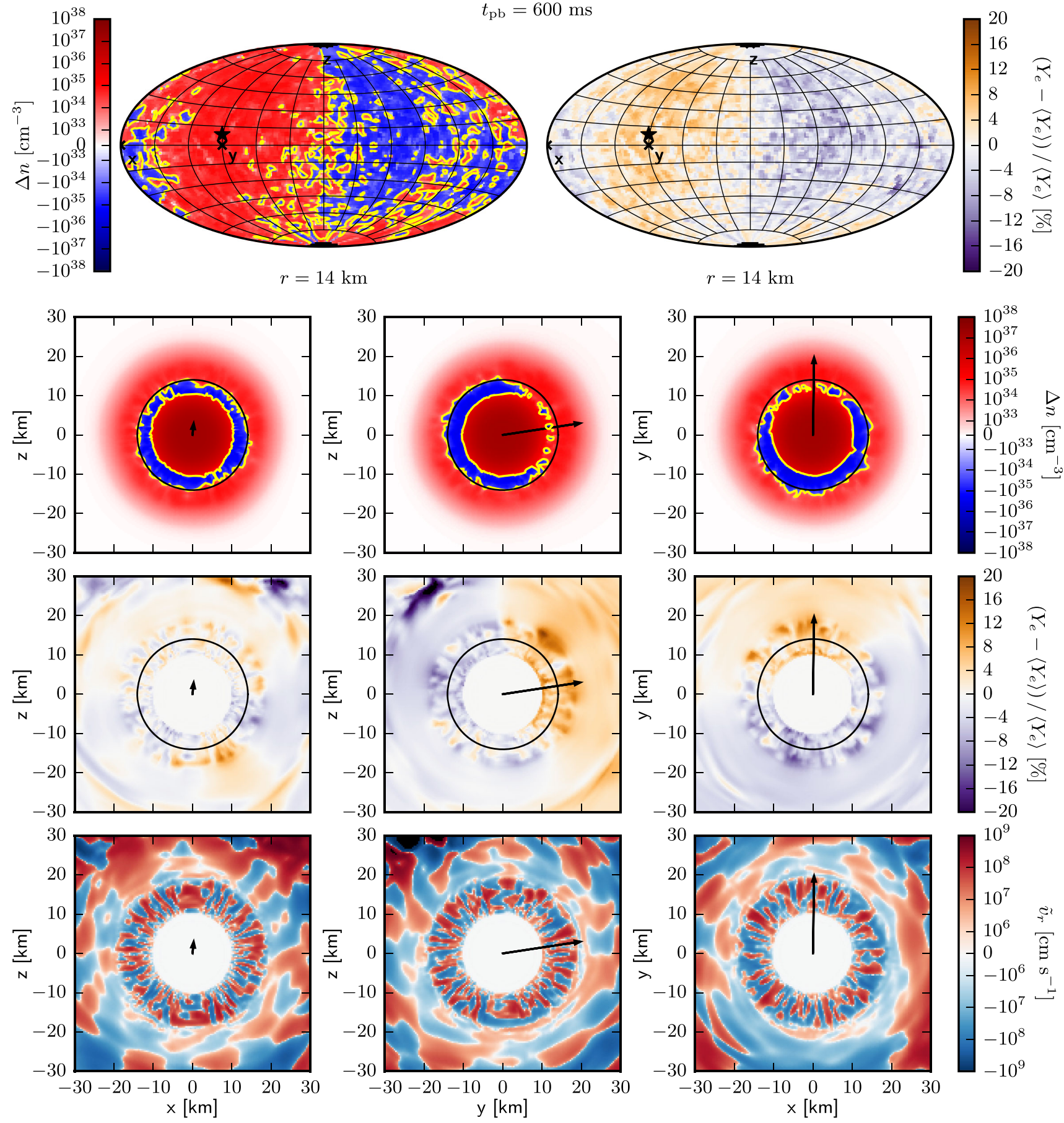

FIG. 7. Same as Fig. 4, but at $600 \mathrm{~ms}$ after core bounce. The LESA dipole is very prominent at this time, corresponding to a clear hemispheric asymmetry of $Y_{e}$ in the convective shell inside the PNS and the overlying outer PNS layers. Volumes with $\Delta n_{\nu}<0$ and flavor-unstable boundaries are concentrated mostly in the hemisphere pointing opposite to the LESA dipole vector (which is indicated by black asterisks and arrows).

use of the diffusion approximation to express the leptonnumber flux, $\Delta F_{\nu}^{r}=F_{\nu_{e}}^{r}-F_{\bar{\nu}_{e}}^{r}$ through $F_{\nu_{i}}^{r}=-D_{\nu_{i}} \partial n_{\nu_{i}} / \partial r$, where $D_{\nu_{i}}=\frac{1}{3} c \lambda_{\nu_{i}}$ is the diffusion coefficient and $\lambda_{\nu_{i}}$ the (energy-averaged) mean free path. Introducing a mean free path $\bar{\lambda}$ suitably averaged between $\nu_{e}$ and $\bar{\nu}_{e}$, we can write for the effective neutrino-lepton number flux in the diffusion regime:

$$
\Delta F_{\nu}^{r}=-\frac{1}{3} c \bar{\lambda} \frac{\partial\left(\Delta n_{\nu}\right)}{\partial r} .
$$



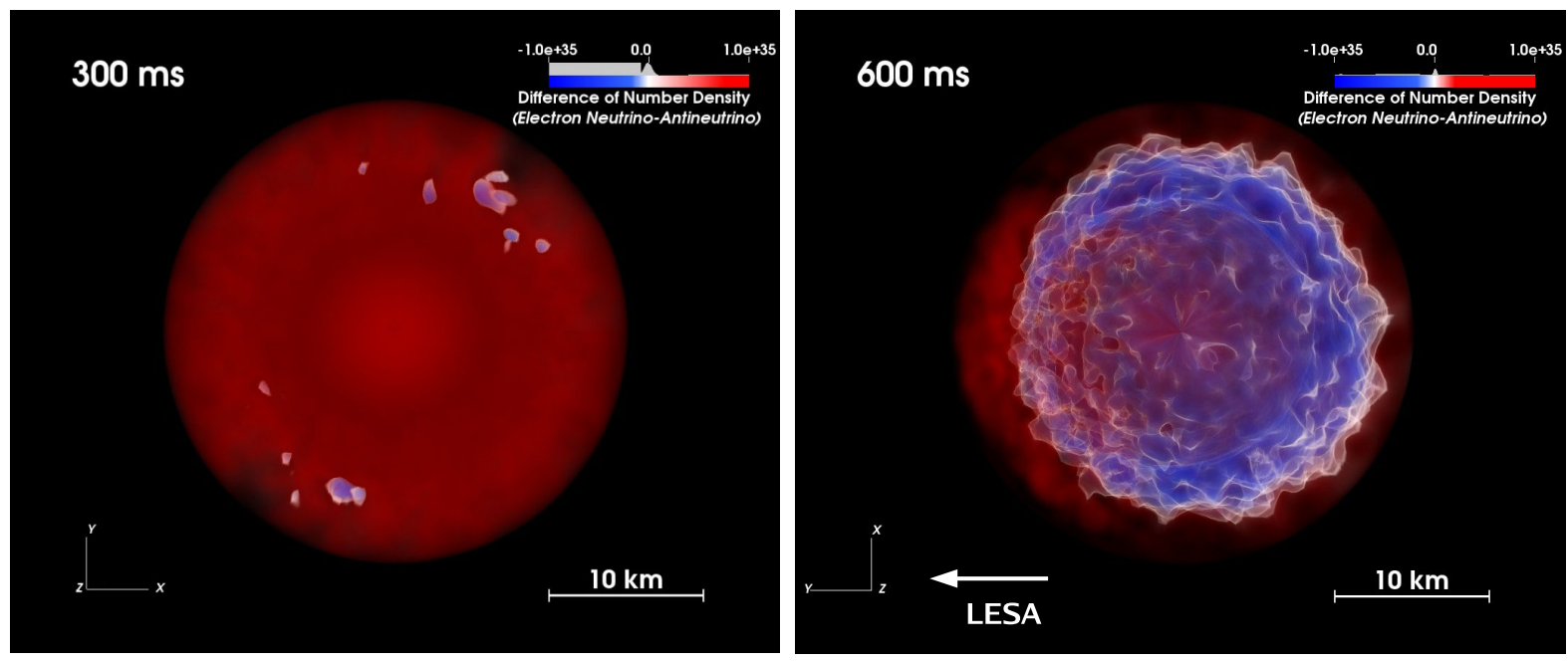

FIG. 8. 3D volume renderings of $\Delta n_{\nu}$ in the $9 M_{\odot}$ model at $300 \mathrm{~ms}$ after bounce (left) and $600 \mathrm{~ms}$ after bounce (right). Red hues indicate excess of $\nu_{e}$ and thus positive $\Delta n_{\nu}$, blue hues excess of $\bar{\nu}_{e}$, and therefore negative values of $\Delta n_{\nu}$. Flavor-unstable locations are in between near $\Delta n_{\nu}=0$ (whitish areas). First "raisins" with flavor-unstable skins become visible at about $300 \mathrm{~ms}$, whereas at $600 \mathrm{~ms}$ flavor-unstable locations can be found near a radius of $14 \mathrm{~km}$ in the whole convective layer of the PNS. Note the pronounced hemispheric asymmetry of the flavor-unstable 2D surface, which is connected in the anti-LESA direction and is more perforated in the hemisphere which the LESA dipole vector points to (namely the $+y$ direction, as indicated by the white arrow next to the tripod).

The requirement for flavor instability, $\mathcal{F}<0$ [Eqs. (13) and (18)], implies that $\left|\Delta F_{\nu}^{r}\right|>\frac{2}{3}\left|\Delta n_{\nu}\right| c$, or, using Eq. (19), that the radial scale height of changes of the lepton-number density must be smaller than $\frac{1}{2} \bar{\lambda}$ :

$$
\left|\Delta n_{\nu}\left(\frac{\partial\left(\Delta n_{\nu}\right)}{\partial r}\right)^{-1}\right|<\frac{1}{2} \bar{\lambda}
$$

This means that the thickness of the skins of flavor instability is only a fraction of the $\nu_{e}-\bar{\nu}_{e}$-averaged mean free path $\bar{\lambda}$, which is on the order of meters in the PNS region of relevance. This skin thickness is 1 to 2 orders of magnitude below the spatial resolution of the best $2 \mathrm{D}$ and 3D simulations, which explains why a direct evaluation of the flavor-instability criterion on the discrete grid points of the computational mesh can find, basically incidentally, only very few locations of ELN crossings.

Around $300 \mathrm{~ms}$ the first spots of flavor instability can be seen near the radius of $14 \mathrm{~km}$. However, while some moments earlier a small number of individual, isolated points may have fulfilled the instability condition $\mathcal{F}<0$, at $300 \mathrm{~ms}$ these points have already grown to $2 \mathrm{D}$ surfaces enclosing noticeable volumes where $\Delta n_{\nu}<0$. Such raisinlike inclusions are concentrated around regions where $Y_{e}$ is $10 \%-15 \%$ lower than the average. At $400 \mathrm{~ms}$ the $\Delta n_{\nu}<0$ volumes have considerably grown and partly merged, enveloped by a coherent surface, besides still existing smaller droplets. This trend continues until our last displayed snapshot at $600 \mathrm{~ms}$. It is obvious that by this time the quadrupole-dominated pattern of concentrations of instability regions that characterizes the situation at $400 \mathrm{~ms}$ and $500 \mathrm{~ms}$ has evolved to a distribution that is clearly dominated by a prominent dipolar asymmetry. While in one hemisphere there are extended blue 3D regions with $\Delta n_{\nu}<0$, the opposite hemisphere still exhibits only scattered spots where this condition is fulfilled. As time goes on these blue regions grow along with a decreasing electron fraction and rising density because the PNS gradually deleptonizes and contracts. It is obvious that the large-scale quadrupolar and dipolar asymmetries of these regions correlate with such asymmetries in the relative variations of $Y_{e}$.

Figure 8 visualizes by 3D volume rendering the situation in the $9 M_{\odot}$ model at the postbounce time of $t=300 \mathrm{~ms}$, when the first scattered "raisins" with $\Delta n_{\nu}<0$ inside and flavor-unstable conditions in their skins have grown. This is compared to the situation at $600 \mathrm{~ms}$ when a whole 3D shell between $\sim 10 \mathrm{~km}$ and $\sim 14 \mathrm{~km}$ with $\bar{\nu}_{e}$ excess over $\nu_{e}$ has developed, more widespread on one side of the PNS than on the other one. This hemispheric asymmetry is connected to the LESA phenomenon and establishes in correlation with the growing dipole amplitude of the LESA, which can be seen in the Aitoff projections of the normalized leptonnumber flux, $\Delta F_{\nu} /\left\langle\Delta F_{\nu}\right\rangle=\left(F_{\nu_{e}}-F_{\bar{\nu}_{e}}\right) /\left\langle\Delta F_{\nu}\right\rangle$ (evaluated far outside the PNS at $r=400 \mathrm{~km}$; Fig. 9), and of the $Y_{e}$ distribution inside the PNS at $r=14 \mathrm{~km}$ (Figs. 4-7).

The black asterisks in the Aitoff plots in all of these figures and the black arrows in the cross-sectional cuts of Figs. 4-7 mark the dipole directions of the lepton-number flux. These markers are missing in the plots for $t=300 \mathrm{~ms}$, because at that early time the emission dipole is not well developed. But once it is present in a clear way, the dipole 

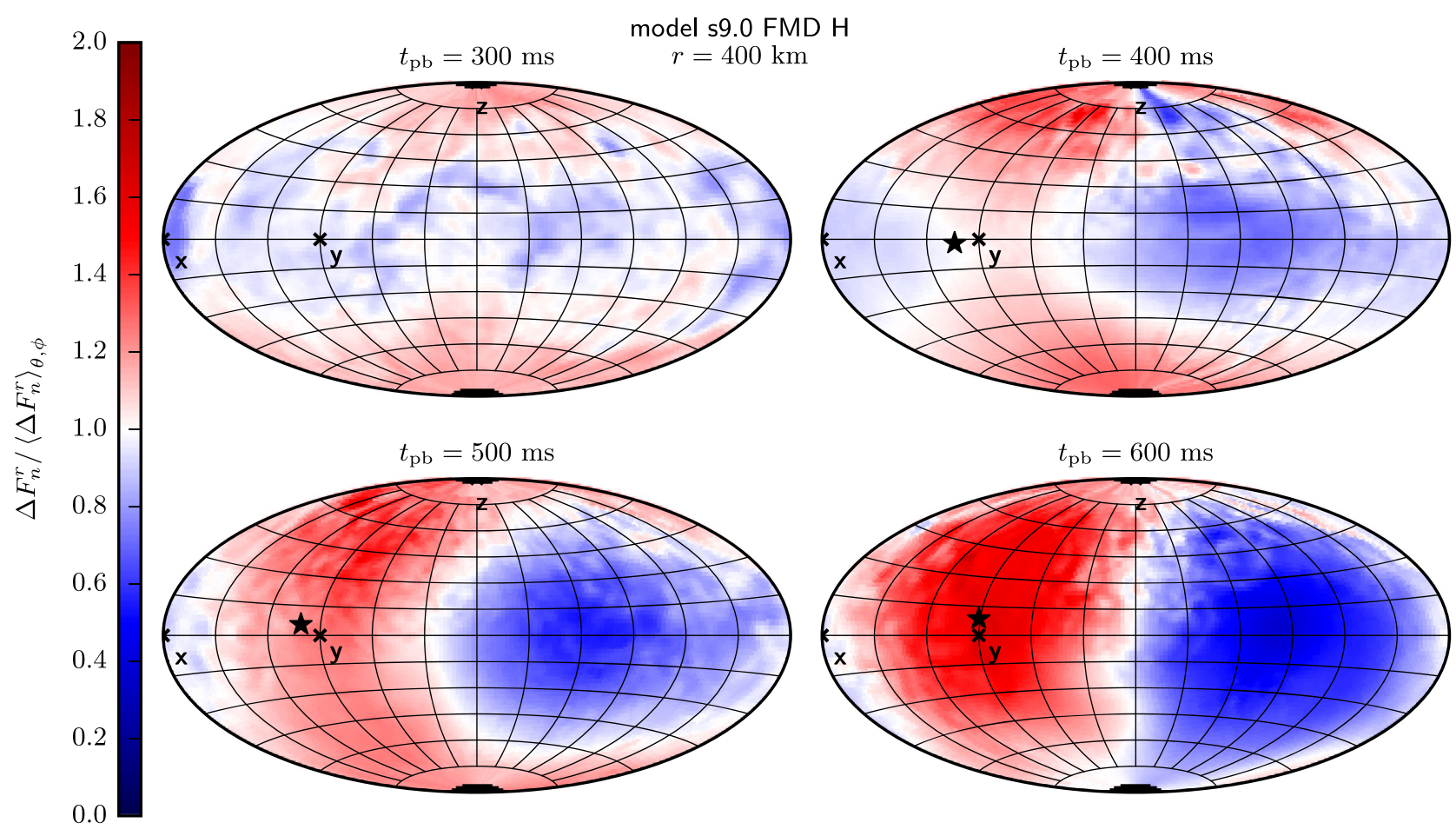

FIG. 9. Aitoff projections of the color-coded electron lepton-number flux, $\Delta F_{\nu}=F_{\nu_{e}}-F_{\bar{\nu}_{e}}$, normalized to the angle-averaged value at a radius of $400 \mathrm{~km}$ in the $9 M_{\odot}$ model at 300,400, 500, and $600 \mathrm{~ms}$ after core bounce (panels from top left to bottom right). The development of a pronounced LESA dipole at $t>300 \mathrm{~ms}$ is obvious, the dipole direction is indicated by black asterisks and is always close to the $+y$ axis of the computational polar grid (see Model s9.0 FMD H in [45]).

direction is extremely stable in the $9 M_{\odot}$ model, varying only very little around the $+y$ direction of the polar coordinate grid of the computation (see Model s9.0 FMD H in Fig. 3 of [45]). It is evident from all these figures that ELN crossings are less favored on the hemisphere in the direction of the LESA dipole vector. As we will discuss in more detail in Sec. IV B, the reason for this observation is the higher electron fraction in the PNS convective shell on this side. As discussed in [45] (see also [30]), convection inside the PNS is stronger in the hemisphere of the LESA dipole direction. This stronger convection transports the electron-lepton number more efficiently out from the edge of the nonconvective central core of the PNS, thus raising the $Y_{e}$ in the convective shell as well as in the overlying near-surface layers of the PNS up to the neutrinospheres.

Figure 10 presents the evolution of the flavor-instability regions in our $20 M_{\odot}$ model by color-coded Aitoff projections and cross-sectional cuts of $\Delta n_{\nu}$ in analogy to Figs. 4-7. The time sequence shows the same basic features as visible in the $9 M_{\odot}$ model, just accelerated because the PNS grows faster in mass due to the higher mass-infall rate in the more massive progenitor. Spots with flavor-unstable conditions are visible already at $170 \mathrm{~ms}$ after bounce near a radius of $14 \mathrm{~km}$ and have grown to large coherent structures at $220 \mathrm{~ms}$. At $270 \mathrm{~ms}$ the regions of $\Delta n_{\nu}<0$ dominate and form a coherent shell around $r=14 \mathrm{~km}$. The very few remaining volumes with $\Delta n_{\nu}>0$ in this shell have essentially disappeared until $370 \mathrm{~ms}$ after bounce. The direction of the LESA emission dipole is indicated by white asterisks in the Aitoff projections and black arrows in the cross-sectional cuts at the times when a strong dipole of the lepton-number emission exists. Again one can notice that flavor-unstable conditions are more widespread in the antiLESA direction, where the electron fraction is lower than in the hemisphere the LESA dipole vector is pointing to. Accordingly, the last islands with $\nu_{e}$ excess (i.e., $\Delta n_{\nu}>0$ ) can be found on this side of the PNS (see panels for $t=270 \mathrm{~ms}$ in Fig. 10).

In the following section we will explain the physical connection between the conditions of flavor instability and the evolution of electron fraction $Y_{e}$, density $\rho$, and temperature inside the PNS in more detail.

\section{B. Properties of ELN crossing points}

Naturally, the relation $\Delta n_{\nu}=n_{\nu_{e}}-n_{\bar{\nu}_{e}} \approx 0$, which we recognized in Sec. III B as a necessary condition for ELN crossings in a regime where neutrinos diffuse and are close to local chemical equilibrium, implies that $\mu_{\nu_{e}}=\mu_{e}+\mu_{p}-$ $\mu_{n} \approx 0$ holds for the electron-neutrino chemical as a function of the chemical potentials of electrons, protons, and neutrons. Only then can $\nu_{e}$ and $\bar{\nu}_{e}$ in local chemical equilibrium have nearly equal number densities. This was pointed out already in Refs. $[35,36]$. 

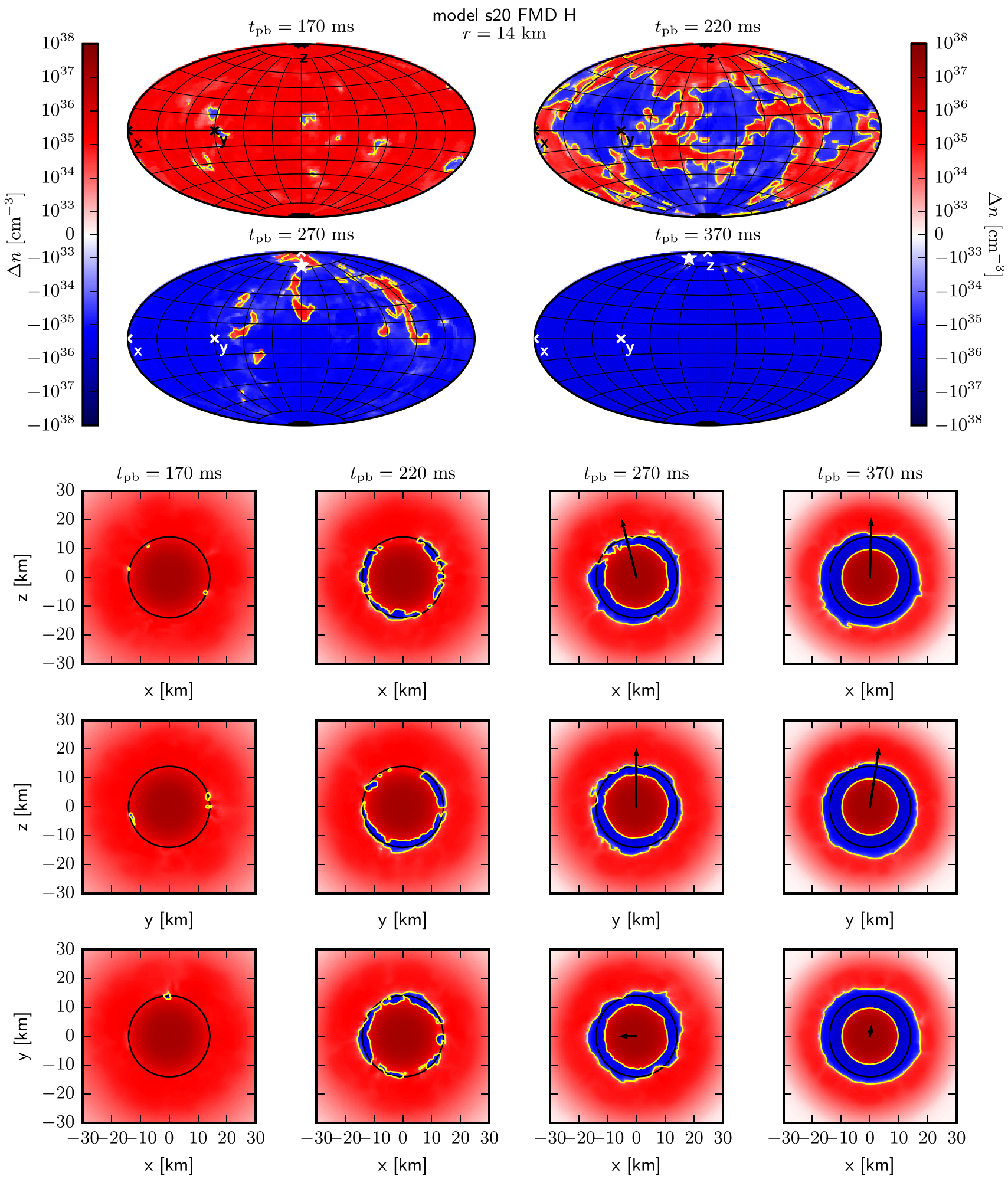

FIG. 10. Time evolution of the regions of fast flavor instability in the $20 M_{\odot}$ model, visualized by Aitoff projections of $\Delta n_{\nu}$ at $14 \mathrm{~km}$ for 170, 220, 270, and $370 \mathrm{~ms}$ after core bounce. Corresponding cross-sectional cuts are also shown with $r=14 \mathrm{~km}$ indicated by a black circle. At $270 \mathrm{~ms}$ and $370 \mathrm{~ms}$ a strong LESA dipole has formed (see Model s20 FMD H in [45]); its vector direction is marked by white asterisks in the Aitoff projections and black arrows in the cuts. Within only $50 \mathrm{~ms}$ initial "raisins" with $\Delta n_{\nu}<0$ (blue) grow to large "pancakes" within a shell around $r=14 \mathrm{~km}$. At $270 \mathrm{~ms}$ nearly the whole shell is included with a few remaining "holes" of $\Delta n_{\nu}>0$ (red) remaining in the hemisphere of the LESA vector direction. At $370 \mathrm{~ms}$ the shell has become wider with no remaining holes. Yellow lines indicate the flavor-unstable locations at the boundaries between regions of $\Delta n_{\nu}<0$ and $\Delta n_{\nu}>0$. 

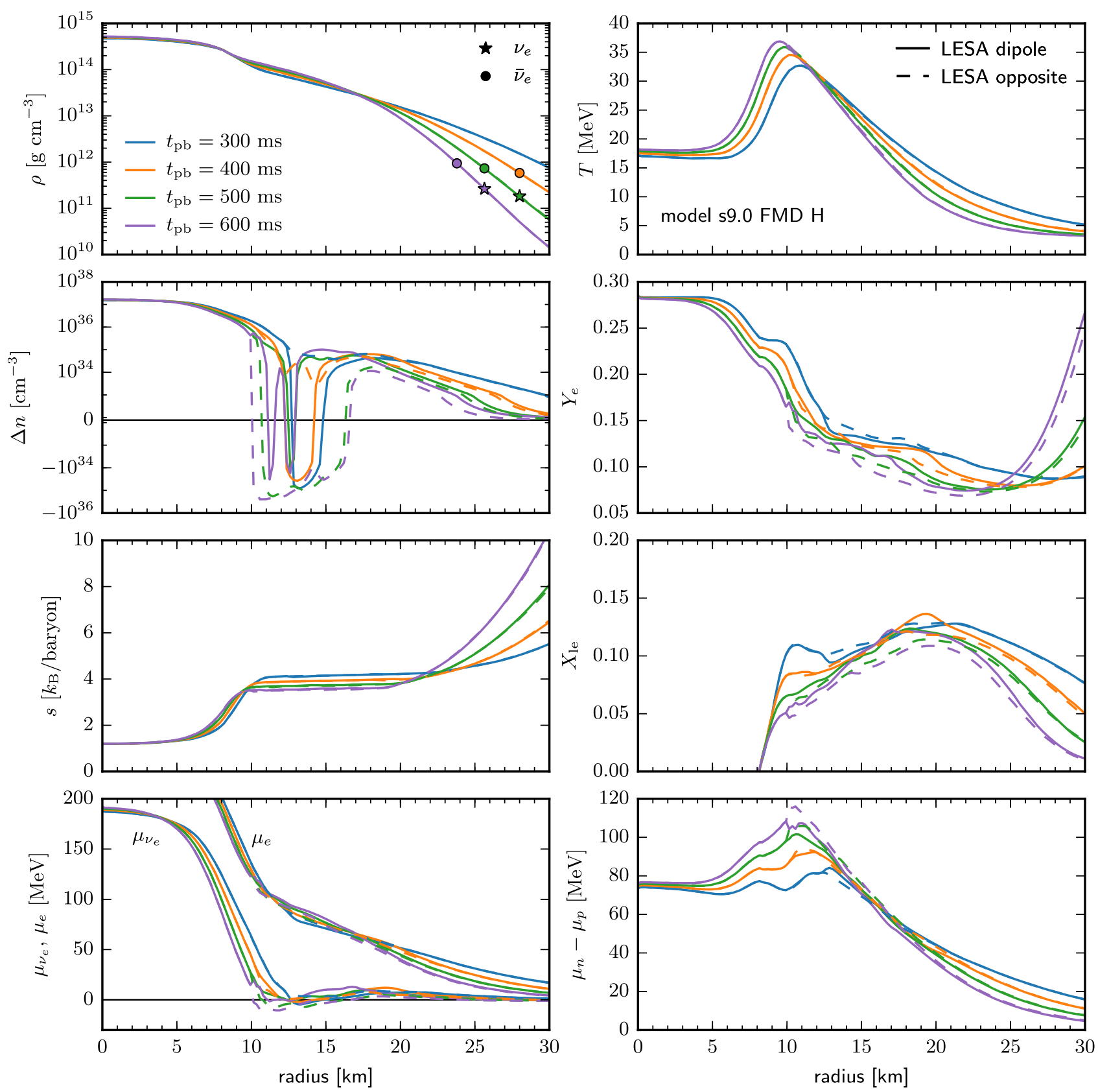

FIG. 11. Radial profiles of mass density ( $\rho$; top left), temperature ( $T$; top right), difference of $\nu_{e}$ and $\bar{\nu}_{e}$ number densities $\left(\Delta n_{\nu}\right.$; second row left), electron fraction ( $Y_{e}$; second row right), gas entropy per nucleon ( $s$; third row left), mass fraction of light nuclei, including $\alpha$ particles, that exist besides free neutrons and protons $\left(X_{\mathrm{le}}\right.$; third row right), chemical potentials of electrons and electron neutrinos $\left(\mu_{e}, \mu_{\nu_{e}}\right.$; bottom left), and chemical potential difference between neutrons and protons $\left(\mu_{n}-\mu_{p}\right.$; bottom right) in our $9 M_{\odot}$ model at 300 , 400, 500, and $600 \mathrm{~ms}$ after core bounce. Solid lines display the profiles in the direction of the LESA dipole, dashed lines in the opposite direction. At $300 \mathrm{~ms}$, when the LESA dipole is still very weak, the later direction of the LESA dipole vector is chosen, because it stably remains in the close vicinity of the $+y$ axis of the computational polar grid once it has developed in this model (see Model s9.0 FMD H in Fig. 3 of [45]). On the density profiles the locations of the neutrinospheres of $\nu_{e}$ and $\bar{\nu}_{e}$ (defined by a spectrally averaged total optical depth of 1) are marked by an asterisk or bullet, respectively. The convective shell inside the PNS can be recognized by the region where the entropy profile is flat.

But what is the reason why fast flavor unstable conditions develop in an increasing volume in the newborn NS? The relation of the chemical potentials that determines $\mu_{\nu_{e}}$ suggests that low $Y_{e}$ and correspondingly low $\mu_{e}$ might allow $\mu_{\nu_{e}}$ to drop to zero and even to negative values.

Figure 11 displays radial profiles of these and other quantities in the LESA and anti-LESA directions of the 
$9 M_{\odot}$ model at four postbounce times. Inspecting the different panels shows that the locations where fast flavor instability is possible $\left(\Delta n_{\nu} \approx 0\right)$ lie within the convective shell in the interior of the PNS; the convective layer can be recognized by the convectively flattened gradient of the entropy per nucleon, $s$. However, neither $Y_{e}$ nor $\mu_{e}$ reach a local minimum where the electron-neutrino chemical potential develops a trough near zero or below, and also the abundances of $\alpha$ particles or light elements up to ${ }^{4} \mathrm{He}$ are not particularly abundant in general in the regions where ELN crossings are favored, in contradiction to arguments in [35]. This can be directly concluded from a comparison of the LESA and anti-LESA directions in Fig. 11. In the hemisphere opposite to the LESA dipole vector, $Y_{e}$ as well as the abundances of $\alpha$ particles and light elements are lower than in the hemisphere the LESA vector points to. Nevertheless, flavor unstable locations are more widespread in the anti-LESA direction at late times (500, $600 \mathrm{~ms}$ after bounce) when the dipole is strong (as discussed in Sec. IVA). Lower values of $Y_{e}$ enhance the possibility of flavor instability, but they are not causal in the first place.

Instead of being linked to peculiarities in the $Y_{e}$ profile, the $\mu_{\nu_{e}}$ trough is found in a region where $\hat{\mu} \equiv \mu_{n}-\mu_{p}$ possesses a local maximum, because high values of $\hat{\mu}$ reduce $\mu_{\nu_{e}}=\mu_{e}-\hat{\mu}$ compared to $\mu_{e}$. The local maximum of $\hat{\mu}$ again correlates tightly with a local temperature maximum, which is a relic of shock heating by the initial propagation of the strong shock front formed at the moment of core bounce. The close connection between maxima of $\hat{\mu}$ and $T$ can easily be understood from considering nondegenerate, nonrelativistic, and noninteracting neutrons and protons as an approximation of nucleons in the subnuclear regime. For such Boltzmann gases the chemical potential of particle species $i=n, p$ with number density $n_{i}$ and rest-mass $m_{i}$ is given by $\mu_{i}=m_{i} c^{2}+$ $k_{\mathrm{B}} T \ln \left(\Lambda_{\mathrm{th}, i}^{3} n_{i} / 2\right)$, where $\Lambda_{\mathrm{th}, i}=h\left(2 \pi m_{i} k_{\mathrm{B}} T\right)^{-1 / 2}$ is the thermal wavelength, $k_{\mathrm{B}}$ the Boltzmann constant, and $h$ the Planck constant. With $Q \equiv\left(m_{n}-m_{p}\right) c^{2}$ one therefore gets

$$
\hat{\mu} \equiv \mu_{n}-\mu_{p}=Q+k_{\mathrm{B}} T \ln \left[\frac{n_{n}}{n_{p}}\left(\frac{m_{p}}{m_{n}}\right)^{3 / 2}\right] .
$$

This relation explains the direct dependence of $\hat{\mu}$ on the plasma temperature, and it also implies that lower $Y_{e}$ in the hemisphere opposite to the LESA vector reduce $\mu_{\nu_{e}}$ not only through lower values of $\mu_{e}$ but also through higher ratios of $n_{n} / n_{p}{ }^{4}$ Non-negligible effects due to nucleon

\footnotetext{
${ }^{4}$ We note that the logarithmic dependence of $\hat{\mu}$ on $n_{n} / n_{p}$ is weak, but also $\mu_{e}$ depends logarithmically on $n_{e}$ when electrons are nondegenerate, and $\mu_{e}$ is proportional to $n_{e}^{1 / 3}$ when electrons are extremely degenerate.
}

interactions in the density regime of interest between some $10^{13} \mathrm{~g} \mathrm{~cm}^{-3}$ and $\sim 10^{14} \mathrm{~g} \mathrm{~cm}^{-3}$ may lead to quantitative changes but do not qualitatively affect this argument.

The development of regions with negative electronneutrino chemical potential, $\mu_{\nu_{e}}<0$, inside the PNS does not only depend on temperature and $Y_{e}$ but also on density. This can be concluded from Fig. 12, where the evolution of the $9 M_{\odot}$ and $20 M_{\odot}$ models is compared. The upper panel displays the growth of the volume of regions with $\mu_{\nu_{e}}<0$. Vertical black lines mark the two earliest instants displayed for both simulations in Fig. 4 and Fig. 10, respectively. The solid lines represent angular averages of the quantities over the whole sphere at $r=14 \mathrm{~km}$, and the crosses indicate the conditions inside the (still very small) volumes that fulfill $\Delta n_{\nu}<0$ at $300 \mathrm{~ms}(170 \mathrm{~ms})$ in the $9 M_{\odot}\left(20 M_{\odot}\right)$ model (the two instants marked by the vertical black lines). Since the conditions in the $\mu_{\nu_{e}}<0$ volumes are special and for some quantities tend to be extreme compared to the conditions on the rest of the sphere, the crosses do not lie on the curves of the angular averages. ${ }^{5}$

The crosses in the second panel of Fig. 12 show that at the marked instants the electron-neutrino chemical potential begins to become negative in the raisinlike spots that are visible in the Aitoff projections of the two models (Figs. 4 and 10), whereas the angle-averaged values of $\mu_{\nu_{e}}$ at $r=$ $14 \mathrm{~km}$ are still somewhat higher, with a monotonically decreasing trend with time. The remaining three panels contain the evolution of density, electron fraction, and temperature, again angle averaged at $r=14 \mathrm{~km}$. In both models flavor-unstable raisin skins begin to occur when the density reaches roughly $5 \times 10^{13} \mathrm{~g} \mathrm{~cm}^{-3}$, whereas $Y_{e}$ and temperature are different between the two models at this time.

From Fig. 13 it becomes clear where these differences originate from. The plot shows a set of isocontours for different, fixed values of the electron fraction (color coded) in the density-temperature plane. All contours are defined by the condition $\mu_{\nu_{e}}=0$. At each point $(\rho, T)$ this condition is fulfilled only for a single value of the electron fraction. Each $\mu_{\nu_{e}}=0$ contour encloses a $\rho-T$ domain where $\mu_{\nu_{e}}>0$ for the $Y_{e}$ value of the contour, whereas $\mu_{\nu_{e}}<0$ holds for the same $Y_{e}$ value outside of this $\mu_{\nu_{e}}=0$ contour (see also Figs. 11 and 12 in Ref. [51]).

In the inset of Fig. 13, the evolution tracks of both SN models in the $\rho-T-Y_{e}$ space, with all quantities angle averaged at $r=14 \mathrm{~km}$, are superimposed on the contours of vanishing neutrino chemical potential. The tracks start at low values of temperature and density with a high value of the electron fraction, and evolve from the lower left to the upper right of the inset as the PNS contracts and heats up by compression and conversion of electron degeneracy energy

\footnotetext{
${ }^{5}$ The crosses correspond to data at $r=14 \mathrm{~km}$ and $(\theta, \phi)=$ $\left(109.00^{\circ}, 57.375^{\circ}\right)$ for the $9 M_{\odot}$ model and $(\theta, \phi)=\left(75.00^{\circ}\right.$, $\left.86.625^{\circ}\right)$ for the $20 M_{\odot}$ case.
} 

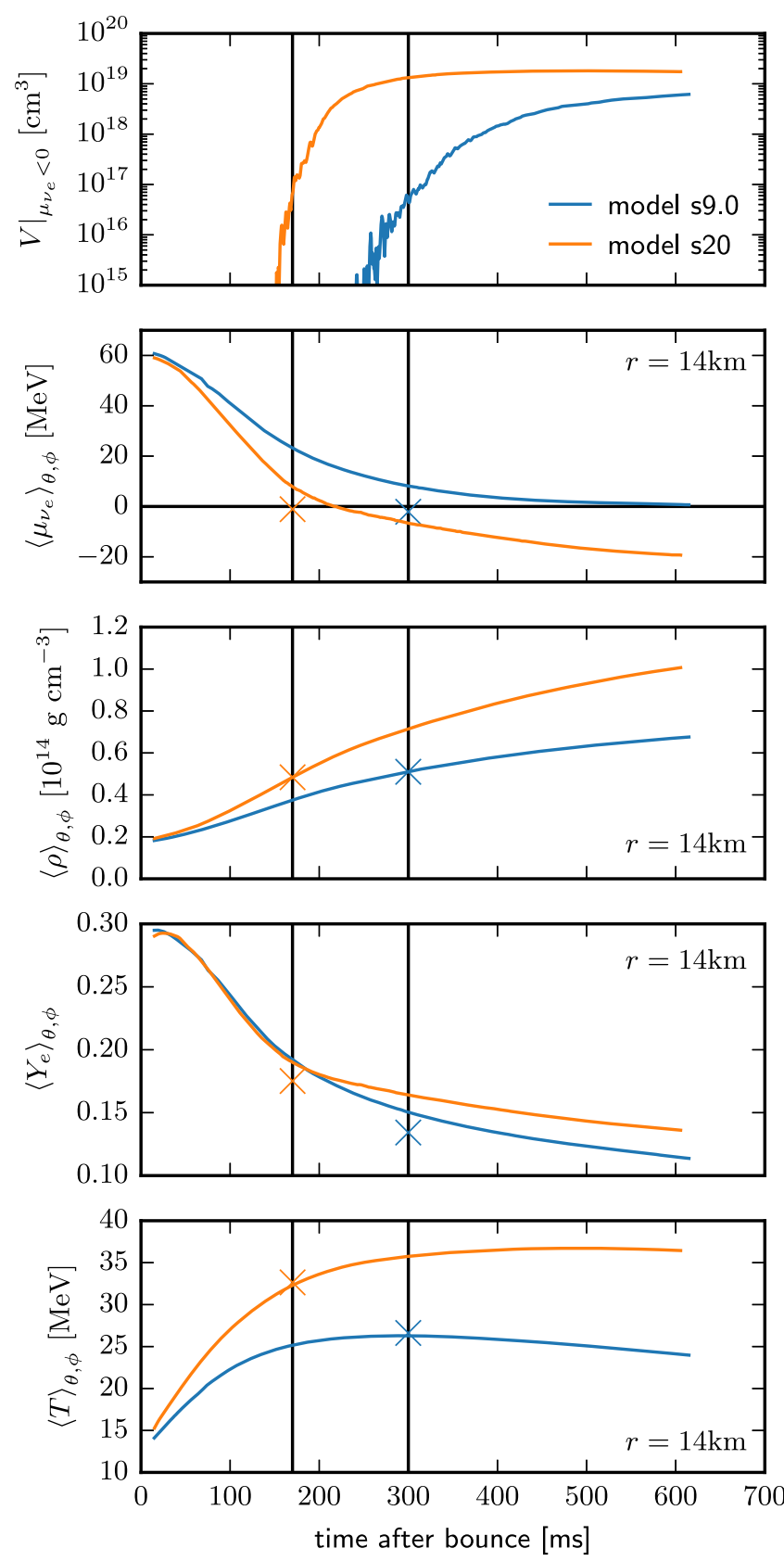

FIG. 12. Time-dependent comparison of the $9 M_{\odot}$ and $20 M_{\odot}$ models with respect to the development of flavor-unstable skins around regions of $\Delta n_{\nu}<0$, measured by the spatial volumes $\left.V\right|_{\mu_{\nu_{e}}<0}$ enclosed by these boundary layers (top panel). Also displayed is the evolution of quantities that are relevant for understanding this effect: electron-neutrino chemical potential ( $\mu_{\nu_{e}}$; second panel), mass density ( $\rho$; third panel), electron fraction $\left(Y_{e}\right.$; fourth panel), and gas temperature $(T$; bottom panel), all angle averaged at our representative radius of $r=14 \mathrm{~km}$. The two vertical black lines mark the earliest moments of the evolution of both models $(300 \mathrm{~ms}$ and $170 \mathrm{~ms}$ after bounce, respectively) shown in Figs. 4 and 10. The crosses indicate the conditions that are found inside the raisinlike volumes with $\Delta n_{\nu}<0$ at these early times. Some of these conditions tend to be extreme compared to the angular averages, for which reason the crosses do not lie on the curves.

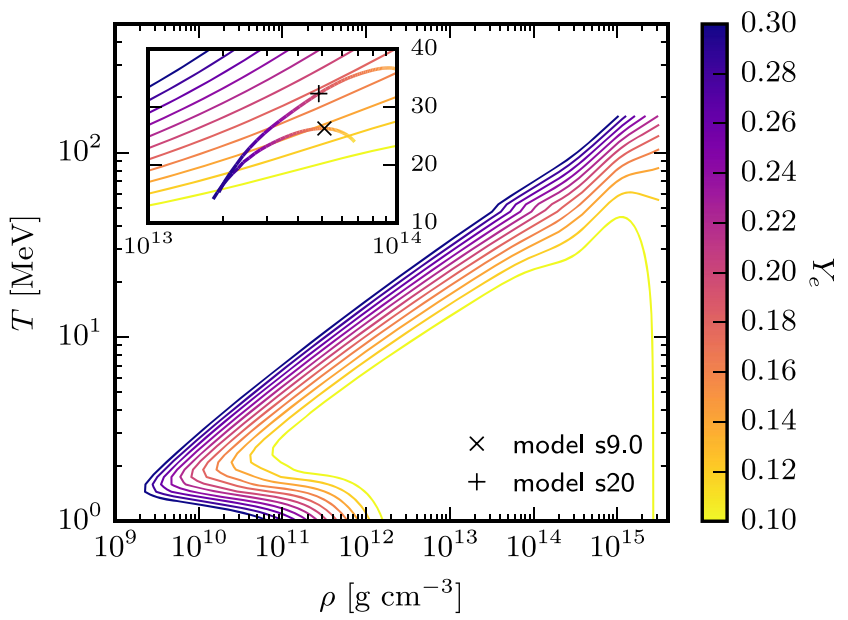

FIG. 13. Contours of vanishing neutrino chemical potential $\left(\mu_{\nu_{e}}=0\right)$ in the temperature-density plane for different constant values of $Y_{e}$ (color coded), evaluated for the nuclear equation of state employed in our 3D SN simulations (SFHo from [52]). The inset shows a zoom of the density interval between $10^{13} \mathrm{~g} \mathrm{~cm}^{-3}$ and $10^{14} \mathrm{~g} \mathrm{~cm}^{-3}$ with the evolution paths of the $9 M_{\odot}$ and $20 M_{\odot}$ models superimposed, also color coded for the evolving value of $\left\langle Y_{e}\right\rangle$, angle averaged at a radius of $14 \mathrm{~km}$ in each model. Along the tracks $\left\langle Y_{e}\right\rangle$ decreases as the PNS deleptonizes; the evolution therefore proceeds from the lower left to the upper right. Spatial locations of flavor-instability begin to appear when $\left\langle Y_{e}\right\rangle$ on the track becomes equal to the $Y_{e}$ of a $\mu_{\nu_{e}}=0$ contour, which is visible by the color of the evolution track matching the color of the contour. The corresponding instants are approximately marked by symbols, corresponding to the times of the vertical lines in Fig. 12 (the cross belongs to the $9 M_{\odot}$ model, the plus to the $20 M_{\odot}$ simulation).

to thermal energy (Joule heating). ELN crossings become possible when the electron fraction on the track matches the $Y_{e}$ value of one of the contours, i.e., when the color of the track and of a contour are the same. The symbols mark the $(\rho, T)$ locations when first noticeable flavor-unstable spots occur in the two SN runs at $r=14 \mathrm{~km}$ (corresponding to the two vertical lines in Fig. 12).

In the $20 M_{\odot}$ model the mass of the PNS grows faster than in the $9 M_{\odot}$ simulation because of the bigger massaccretion rate in the more massive progenitor. Therefore the temperature in the PNS interior increases more rapidly and more steeply with density, and reaches greater values, allowing for a match of the electron fraction with one of the $\mu_{\nu_{e}}=0$ isocontours more quickly and at a higher value of $Y_{e}$. As a consequence, fast flavor unstable conditions occur earlier in the $20 M_{\odot}$ model than in the $9 M_{\odot}$ case.

Compressional and Joule heating and continuous deleptonization are characteristic features of the neutrinocooling evolution of newborn NSs. Our study includes a low-mass NS of a $9 M_{\odot}$ progenitor as well as a more massive NS in the $20 M_{\odot}$ model. Both of them develop flavor-unstable conditions in an increasing volume of the convective layer (Fig. 12), and the rising temperature 
naturally leads to a match of the decreasing $Y_{e}$ values along evolution tracks with the $Y_{e}$ of isocontours for $\mu_{\nu_{e}}=0$ (Fig. 13). We therefore expect that fast flavor unstable regions in the deep interior of PNSs are a generic phenomenon during PNS cooling.

\section{CONCLUSIONS}

We performed a detailed investigation of 3D state-of-theart SN models for the presence of fast neutrino flavor instability as well as to study the favorable conditions. These fast conversions are associated with crossings in the angular distribution of the ELN. However, the simulations we analyzed did not provide the detailed neutrino angular distributions. To overcome this limitation, we adopted a novel method for calculating the growth rate of the instability, which is based only on the angular moments of the ELN up to second order [47]. We applied our analysis to SN simulations of $9 M_{\odot}$ and $20 M_{\odot}$ progenitors, which were recently conducted by the Garching group $[45,46]$. In both models we found conditions for fast flavor instability deep inside the PNS in a radial range of $10 \mathrm{~km} \lesssim r \lesssim 20 \mathrm{~km}$, where all flavors of neutrinos are in the diffusive regime and close to local chemical equilibrium.

We thus confirm similar recent detections of locations of ELN crossings in the PNS interior based on Boltzmann transport results in 2D time-dependent and 2D/3D fixedbackground SN models in Refs. [35,36]. However, we showed that the direct evaluation of flavor-instability conditions with the discretized output of numerical simulations leads to the identification of only a few individual, isolated points of ELN crossings. We argued that this finding on grounds of our method, and most likely also by the approaches used in the previous analyses, is a numerical artifact and misses the full phenomenology of the physical effects.

In reality the spatial locations of fast flavor instability are extended, narrow boundary layers surrounding volumes in $3 \mathrm{D}$ space where the $\bar{\nu}_{e}$ number density exceeds the $\nu_{e}$ number density. These surface layers have a thickness of roughly a neutrino mean free path and they grow from the skins of initially scattered, raisinlike inclusions to the envelopes of increasingly larger regions, until they finally form the inner and outer surfaces of a closed layer with $n_{\bar{\nu}_{e}}>n_{\nu_{e}}$.

The region where this happens is located within the convective layer of the PNS where, on the one hand, convective transport of the lepton number causes a rapid decline of the electron fraction, and, on the other hand, a local temperature maximum is further increased when the PNS contracts and compression as well as the conversion of electron-degeneracy energy to thermal energy heat the stellar plasma. Rising density and temperature combined with a decreasing electron fraction naturally drive this layer in the PNS toward conditions where the electron-neutrino chemical potential is $\mu_{\nu_{e}} \leq 0$, implying an excess of $\bar{\nu}_{e}$ relative to $\nu_{e}$. Since the lepton-number emission dipole associated with the LESA phenomenon is caused by stronger PNS convection in the hemisphere of the dipole direction, conditions for ELN crossings are more widespread in the opposite hemisphere, where PNS convection is weaker and therefore the electron fraction is not efficiently replenished by leptons carried outward from the edge of the nonconvective central core.

Our result backs the findings in $[35,36]$ and points to ELN crossings in the PNS convection layer as a generic phenomenon during the cooling evolution of newborn NSs. In contrast to these previous works we have demonstrated that the regions of fast flavor unstable conditions are not fluctuating and pointlike, but instead they are large-scale and long-lasting spatial structures.

This opens new directions of research. Presently, however, it is unclear whether fast flavor conversions in the deep interior of the PNS can have major consequences for the PNS cooling and/or SN evolution. The instability takes place in an extremely thin layer where $\mu_{\nu_{e}}$ is close to zero. Since these locations are deep inside the neutrinospheres of all neutrino species, neutrinos of all flavors are very close to chemical equilibrium. Therefore $\mu_{\nu_{e}} \approx 0$ implies that $\nu_{e}$ and $\bar{\nu}_{e}$ possess phase-space distributions that are very similar not only to each other but also to those of muon and tau neutrinos. At such conditions flavor exchange might have little impact on the overall conditions. Given the importance of potential effects, our work should stimulate further investigations. In view of the mutual support between our results and those obtained in Refs. [35,36], we conclude that our method based on angular moments is well suitable to analyze SN models computed with neutrino transport schemes that do not provide the detailed neutrino angular distributions.

The presence of fast conversions adds an additional layer of complexity to SN simulations. The current paradigm of flavor conversions is based on a separation from the treatment of neutrino interactions and transport. However, if fast conversions occur in the region of neutrino spectra formation or even deep inside the PNS, a selfconsistent characterization of both of these phenomena will require new strategies for a simultaneous treatment of flavor oscillations and collisional effects. This will be a formidable task, since fast flavor conversions occur on time and length scales much shorter than usually resolved in global simulations of stellar collapse and explosions. Therefore, new methodical approaches will be needed. It is obvious that a huge amount of work still remains to be done to understand the role of neutrino flavor conversions in the cores of collapsing stars.

\section{ACKNOWLEDGMENTS}

We are grateful to Georg Raffelt for discussions during the development of the project, to Shaoming Zhang for the 
3D visualizations shown in Fig. 8, and to Irene Tamborra for comments on the manuscript. The work of F. C. is partially supported by the Deutsche Forschungsgemeinschaft (DFG, German Research Foundation) through Sonderforschungsbereich (SFB, Collaborative Research Centre) Grant No. SFB-1258 "Neutrinos and Dark Matter in Astro- and Particle Physics (NDM)" and under Germany's Excellence Strategy through Excellence Cluster ORIGINS (EXC-2094)-390783311" as well as by the European Union through Grant No. H2020-MSCA-ITN2015/674896 (Innovative Training Network "Elusives"). M. S. acknowledges support from the National Science Foundation, Grant No. PHY-1630782, and from the Heising-Simons Foundation, Grant No. 2017-228. The work of B. D. is partially supported by the Department of Atomic Energy (Government of India) Research Project No. 12-R\&D-TFR-5.02-0200, the Department of Science and Technology (Government of India) through a Ramanujan Fellowship, and by the Max-PlanckGesellschaft through a Max Planck Partner Group. The work of A. M. is partially supported by the Italian Istituto Nazionale di Fisica Nucleare (INFN) through the "Theoretical Astroparticle Physics" project and by research Grant No. 2017W4HA7S "NAT-NET: Neutrino and Astroparticle Theory Network" under the program PRIN 2017 funded by the Italian Ministero dell'Università e della Ricerca (MUR). At Garching, funding by the European Research Council through Grant ERC-AdG No. 341157COCO2CASA and by the DFG through SFB Grant No. SFB1258 "Neutrinos and Dark Matter in Astro- and Particle Physics (NDM)" and under Germany's Excellence Strategy through Excellence Cluster ORIGINS (EXC-2094)390783311 is acknowledged. The work of G. S. is partially supported by the DFG under Germany's Excellence Strategy through Excellence Cluster "Quantum Universe" (EXC2121) - 390833306. Computer resources for this project have been provided by the Leibniz Supercomputing Centre (LRZ) under LRZ Project ID pr62za and by the Max Planck Computing and Data Facility (MPCDF) on the HPC system Hydra.
[1] J. T. Pantaleone, Neutrino oscillations at high densities, Phys. Lett. B 287, 128 (1992).

[2] V. A. Kostelecky and S. Samuel, Selfmaintained coherent oscillations in dense neutrino gases, Phys. Rev. D 52, 621 (1995).

[3] S. Pastor and G. G. Raffelt, Flavor oscillations in the Supernova Hot Bubble Region: Nonlinear Effects of Neutrino Background, Phys. Rev. Lett. 89, 191101 (2002).

[4] R. F. Sawyer, Speed-up of neutrino transformations in a supernova environment, Phys. Rev. D 72, 045003 (2005).

[5] H. Duan, G. M. Fuller, J. Carlson, and Y.-Z. Qian, Simulation of coherent non-linear neutrino flavor transformation in the supernova environment. 1. Correlated neutrino trajectories, Phys. Rev. D 74, 105014 (2006).

[6] S. Hannestad, G. G. Raffelt, G. Sigl, and Y. Y. Y. Wong, Self-induced conversion in dense neutrino gases: Pendulum in flavour space, Phys. Rev. D 74, 105010 (2006); Erratum, Phys. Rev. D 76, 029901 (2007).

[7] G. L. Fogli, E. Lisi, A. Marrone, and A. Mirizzi, Collective neutrino flavor transitions in supernovae and the role of trajectory averaging, J. Cosmol. Astropart. Phys. 12 (2007) 010.

[8] R. F. Sawyer, The multi-angle instability in dense neutrino systems, Phys. Rev. D 79, 105003 (2009).

[9] B. Dasgupta, A. Dighe, G. G. Raffelt, and A. Yu. Smirnov, Multiple Spectral Splits of Supernova Neutrinos, Phys. Rev. Lett. 103, 051105 (2009).

[10] R. F. Sawyer, Neutrino Cloud Instabilities Just above the Neutrino Sphere of a Supernova, Phys. Rev. Lett. 116, 081101 (2016).

[11] H. Duan, G. M. Fuller, and Y.-Z. Qian, Collective neutrino oscillations, Annu. Rev. Nucl. Part. Sci. 60, 569 (2010).
[12] A. Mirizzi, I. Tamborra, H.-T. Janka, N. Saviano, K. Scholberg, R. Bollig, L. Hüdepohl, and S. Chakraborty, Supernova neutrinos: Production, oscillations and detection, Riv. Nuovo Cimento 39, 1 (2016).

[13] S. Chakraborty, R. Hansen, I. Izaguirre, and G. G. Raffelt, Collective neutrino flavor conversion: Recent developments, Nucl. Phys. B908, 366 (2016).

[14] S. Horiuchi and J. P. Kneller, What can be learned from a future supernova neutrino detection? J. Phys. G 45, 043002 (2018).

[15] S. Chakraborty, R. S. Hansen, I. Izaguirre, and G. G. Raffelt, Self-induced neutrino flavor conversion without flavor mixing, J. Cosmol. Astropart. Phys. 03 (2016) 042.

[16] B. Dasgupta, A. Mirizzi, and M. Sen, Fast neutrino flavor conversions near the supernova core with realistic flavordependent angular distributions, J. Cosmol. Astropart. Phys. 02 (2017) 019.

[17] I. Izaguirre, G. G. Raffelt, and I. Tamborra, Fast Pairwise Conversion of Supernova Neutrinos: A DispersionRelation Approach, Phys. Rev. Lett. 118, 021101 (2017).

[18] F. Capozzi, B. Dasgupta, E. Lisi, A. Marrone, and A. Mirizzi, Fast flavor conversions of supernova neutrinos: Classifying instabilities via dispersion relations, Phys. Rev. D 96, 043016 (2017).

[19] A. Dighe and M. Sen, Nonstandard neutrino selfinteractions in a supernova and fast flavor conversions, Phys. Rev. D 97, 043011 (2018).

[20] B. Dasgupta and M. Sen, Fast neutrino flavor conversion as oscillations in a Quartic potential, Phys. Rev. D 97, 023017 (2018). 
[21] S. Abbar and H. Duan, Fast neutrino flavor conversion: Roles of dense matter and spectrum crossing, Phys. Rev. D 98, 043014 (2018).

[22] S. Airen, F. Capozzi, S. Chakraborty, B. Dasgupta, G. Raffelt, and T. Stirner, Normal-mode analysis for collective neutrino oscillations, J. Cosmol. Astropart. Phys. 12 (2018) 019.

[23] S. Abbar and M. C. Volpe, On fast neutrino flavor conversion modes in the nonlinear regime, Phys. Lett. B 790, 545 (2019).

[24] C. Yi, L. Ma, J. D. Martin, and H. Duan, Dispersion relation of the fast neutrino oscillation wave, Phys. Rev. D 99 , 063005 (2019) 063005.

[25] F. Capozzi, G. Raffelt, and T. Stirner, Fast neutrino flavor conversion: Collective motion vs. decoherence, J. Cosmol. Astropart. Phys. 09 (2019) 002.

[26] S. Shalgar and I. Tamborra, On the occurrence of crossings between the angular distributions of electron neutrinos and antineutrinos in the supernova core, Astrophys. J. 883, 80 (2019).

[27] J. D. Martin, C. Yi, and H. Duan, Dynamic fast flavor oscillation waves in dense neutrino gases, Phys. Lett. B 800, 135088 (2020).

[28] J. D. Martin, J. Carlson, and H. Duan, Spectral swaps in a two-dimensional neutrino ring model, Phys. Rev. D 101, 023007 (2020).

[29] I. Tamborra, L. Hüdepohl, G. Raffelt, and H.-T. Janka, Flavor-dependent neutrino angular distribution in corecollapse supernovae, Astrophys. J. 839, 132 (2017).

[30] I. Tamborra, F. Hanke, H.-T. Janka, B. Müller, G. G. Raffelt, and A. Marek, Self-sustained asymmetry of lepton-number emission: A new phenomenon during the supernova shockaccretion phase in three dimensions, Astrophys. J. 792, 96 (2014).

[31] S. Abbar, H. Duan, K. Sumiyoshi, T. Takiwaki, and M. C. Volpe, On the occurrence of fast neutrino flavor conversions in multidimensional supernova models, Phys. Rev. D 100, 043004 (2019).

[32] M. Delfan Azari, S. Yamada, T. Morinaga, W. Iwakami, H. Okawa, H. Nagakura, and K. Sumiyoshi, Linear analysis of fast-pairwise collective neutrino oscillations in corecollapse supernovae based on the results of Boltzmann simulations, Phys. Rev. D 99, 103011 (2019).

[33] T. Morinaga, H. Nagakura, C. Kato, and S. Yamada, A new possibility of the fast neutrino-flavor conversion in the pre-shock region of core-collapse supernova, arXiv: 1909.13131.

[34] S. Shalgar, I. Padilla-Gay, and I. Tamborra, Neutrino propagation hinders fast pairwise flavor conversions, arXiv: 1911.09110.

[35] M. D. Azari, S. Yamada, T. Morinaga, H. Nagakura, S. Furusawa, A. Harada, H. Okawa, W. Iwakami, and K. Sumiyoshi, Fast collective neutrino oscillations inside the neutrino sphere in core-collapse supernovae, arXiv:1910. 06176.

[36] S. Abbar, H. Duan, K. Sumiyoshi, T. Takiwaki, and M. C. Volpe, Fast neutrino flavor conversion modes in multidimensional core-collapse supernova models: The role of the asymmetric neutrino distributions, arXiv:1911.01983.

[37] S. W. Bruenn et al., The development of explosions in axisymmetric AB INITIO core-collapse supernova simulations of $12-25 M_{\odot}$ stars, Astrophys. J. 818, 123 (2016).

[38] E. P. O'Connor and S. M. Couch, Two dimensional corecollapse supernova explosions aided by general relativity with multidimensional neutrino transport, Astrophys. J. 854, 63 (2018).

[39] H. Nagakura, W. Iwakami, S. Furusawa, H. Okawa, A. Harada, K. Sumiyoshi, S. Yamada, H. Matsufuru, and A. Imakura, Simulations of core-collapse supernovae in spatial axisymmetry with full Boltzmann neutrino transport, Astrophys. J. 854, 136 (2018).

[40] S. Richers, H. Nagakura, C. D. Ott, J. Dolence, K. Sumiyoshi, and S. Yamada, A detailed comparison of multidimensional Boltzmann neutrino transport methods in core-collapse supernovae, Astrophys. J. 847, 133 (2017).

[41] D. Vartanyan, A. Burrows, D. Radice, M. A. Skinner, and J. Dolence, Revival of the fittest: Exploding core-collapse supernovae from 12 to $25 M_{\odot}$, Mon. Not. R. Astron. Soc. 477, 3091 (2018).

[42] O. Just, R. Bollig, H. T. Janka, M. Obergaulinger, R. Glas, and S. Nagataki, Core-collapse supernova simulations in one and two dimensions: Comparison of codes and approximations, Mon. Not. R. Astron. Soc. 481, 4786 (2018).

[43] R. M. Cabezón, K.-C. Pan, M. Liebendörfer, T. Kuroda, K. Ebinger, O. Heinimann, A. Perego, and F.-K. Thielemann, Core-collapse supernovae in the hall of mirrors. A threedimensional code-comparison project, Astron. Astrophys. 619, A118 (2018).

[44] K.-C. Pan, C. Mattes, E. P. O'Connor, S. M. Couch, A. Perego, and A. Arcones, The impact of different neutrino transport methods on multidimensional core-collapse supernova simulations, J. Phys. G 46, 014001 (2019).

[45] R. Glas, H. T. Janka, T. Melson, G. Stockinger, and O. Just, Effects of LESA in three-dimensional supernova simulations with multidimensional and ray-by-ray-plus neutrino transport, Astrophys. J. 881, 36 (2019).

[46] R. Glas, O. Just, H. T. Janka, and M. Obergaulinger, Threedimensional core-collapse supernova simulations with multidimensional neutrino transport compared to the rayby-ray-plus approximation, Astrophys. J. 873, 45 (2019).

[47] B. Dasgupta, A. Mirizzi, and M. Sen, Simple method of diagnosing fast flavor conversions of supernova neutrinos, Phys. Rev. D 98, 103001 (2018).

[48] L. Johns, H. Nagakura, G. M. Fuller, and A. Burrows, Neutrino oscillations in supernovae: Angular moments and fast instabilities, arXiv:1910.05682.

[49] L. M. Murchikova, E. Abdikamalov, and T. Urbatsch, Analytic closures for M1 neutrino transport, Mon. Not. R. Astron. Soc. 469, 1725 (2017).

[50] H. Nagakura, T. Morinaga, C. Kato, and S. Yamada, Fastpairwise collective neutrino oscillations associated with asymmetric neutrino emissions in core-collapse supernova, Astrophys. J. 886, 139 (2019).

[51] M. Ruffert, H. T. Janka, K. Takahashi, and G. Schaefer, Coalescing neutron stars - a step towards physical models. II. Neutrino emission, neutron tori, and gamma-ray bursts, Astron. Astrophys. 319, 122 (1997).

[52] A. W. Steiner, M. Hempel, and T. Fischer, Core-collapse supernova equations of state based on neutron star observations, Astrophys. J. 774, 17 (2013). 